Pacific

Journal of

Mathematics

\title{
DISCRETE BISPECTRAL DARBOUX TRANSFORMATIONS FROM JACOBI OPERATORS
}

F. Alberto Grünbaum and Milen Yakimov 


\title{
DISCRETE BISPECTRAL DARBOUX TRANSFORMATIONS FROM JACOBI OPERATORS
}

\author{
F. Alberto Grünbaum and Milen Yakimov
}

\begin{abstract}
We construct families of bispectral difference operators of the form $a(n) T+b(n)+c(n) T^{-1}$ where $T$ is the shift operator. They are obtained as discrete Darboux transformations from appropriate extensions of Jacobi operators. We conjecture that along with operators previously constructed by Grünbaum, Haine, Horozov and Iliev they exhaust all bispectral regular (i.e., $a(n) \neq 0, c(n) \neq 0, \forall n \in \mathbb{Z}$ ) operators of the form above.
\end{abstract}

\section{Introduction.}

Back in 1929 S. Bochner [6] posed and solved the problem of isolating all families of orthogonal polynomials that are also eigenfunctions of a fixed, but arbitrary, second order differential operator. He found that they were given by what are nowadays called "the classical orthogonal polynomials", i.e., those of Jacobi, Hermite, Laguerre and (the less known) Bessel. Many developments in the last few years which establish rich links between classical function theory at one end and differential algebra at the other, can be seen as the result of looking for answers to questions that are variants of that of Bochner. Some of these developments are alluded to in the rest of the introduction. Before going into details it is probably worth noticing that while the original paper of Bochner poses and solves the problem in a few pages, the extensions that have been considered in the last 15 years or so are still awaiting complete resolution. This paper takes a step in that direction.

The bispectral problem, as originally formulated by Duistermaat and Grünbaum [7], asks for a description of all situations where a pair of differential operators in the variables $x$ and $z$ have a common eigenfunction $\Psi(x, z)$

$$
\begin{aligned}
& L\left(x, \partial_{x}\right) \Psi(x, z)=\lambda(z) \Psi(x, z), \\
& B\left(z, \partial_{z}\right) \Psi(x, z)=\theta(x) \Psi(x, z) .
\end{aligned}
$$

For simplicity we say that $L$, or $B$, or $\Psi$, are bispectral when the situation above holds.

The results in [7] already revealed a number of interesting connections with a variety of topics ranging from the Korteweg-deVries equation to 
the problem of isomonodromic deformations for differential operators with rational coefficients. Later even more unexpected connections with different areas of pure mathematics were found. These include automorphisms and ideal structure of the Weyl algebra in one variable $[\mathbf{5}, \mathbf{3}]$, representations of the $W_{1+\infty}$ algebra [2], Calogero-Moser system [25], Huygens' principle [4], traces of intertwiners for representations of (quantized) simple Lie algebras $[8,9]$ (the last two in the multivariable case).

In [7] all bispectral differential operators $L\left(x, \partial_{x}\right)$ of second order were classified. Notice that if one insists that $B\left(z, \partial_{z}\right)$ should also be of order two then one is necessarily dealing with the Bessel or Airy cases. In that paper very explicit use was made of the Darboux transformation mapping a given second order differential operator into another one. When starting from an appropriate bispectral $L\left(x, \partial_{x}\right)$ this was shown to produce another such, with a different $B\left(z, \partial_{z}\right)$. Wilson [24] approached the problem from the viewpoint of commutative algebras of differential operators. He classified all maximal bispectral algebras of rank one (which by definition is the greatest common divisor of the orders of all operators in the algebra). In $[\mathbf{1}, \mathbf{1 8}]$ the idea of applying Darboux transformations to commutative algebras of differential operators was developed. This allowed for a unification of the apparently unrelated methods in $[\mathbf{7}, \mathbf{2 4}]$ and an extension of them to the higher rank case. Further interesting results in this direction were obtained in $[\mathbf{1 7}]$.

Grünbaum and Haine considered [10] a discrete-differential version of the above problem when the variable $x$ runs over the integer lattice $\mathbb{Z}$ and accordingly one replaces the differential operator $L\left(x, \partial_{x}\right)$ by a difference operator

$$
L(n, T)=\sum_{i=p}^{q} b_{i}(n) T^{i}, \quad b_{p}(n), b_{q}(n) \not \equiv 0
$$

acting on a function $f(n): \mathbb{Z} \rightarrow \mathbb{C}$ by

$$
(L f)(n)=\sum_{i=p}^{q} b_{i}(n) f(n+i) .
$$

Following $[\mathbf{2 3}, \mathbf{2 2}]$, we define the support of $L(n, T)$ to be the ordered pair $[p, q]$. Such a difference operator will be called regular if the first and the last coefficients $b_{q}(n)$ and $b_{p}(n)$ are nowhere vanishing functions on $\mathbb{Z}$.

As indicated above, this problem is a generalization of the problem of classifying orthogonal polynomials which are eigenfunctions of differential operators. The point is that the standard three term recursion relation gives rise to a very special type of difference operator, represented by a semiinfinite tridiagonal matrix. In [10], Grünbaum and Haine showed that all instances of difference operators with support $[-1,1]$ and second order 
differential operators satisfying (1.1)-(1.2) result by replacing the variable $n$ in the classical cases of the Hermite, Laguerre, Jacobi, and Bessel polynomials (discovered by Bochner, see [6]) by a variable $n+\varepsilon$ with $n$ running over the integer lattice and $\varepsilon$ arbitrary (see Section 2.2 for precise definitions). The differential operator in $z$ is the celebrated hypergeometric second order differential operator of Gauss. It is worth noting that the corresponding eigenfunctions $\Psi(n, z)$ are no longer polynomials. For a recent survey of this area, see [14].

Recently Haine and Iliev considered the classification problem for maximal bispectral difference algebras of rank one [15]. Their treatment is a beautiful extension of Wilson's work [24] where the Grassmannians associated to Darboux transformations on differential operators are substituted with flag varieties coming from such transformations on difference operators. Among these some algebras that contain an operator with support $[-1,1]$ were isolated in [16], where they were conjectured to be all of this type.

The aim of this paper is to make progress in obtaining a discrete-continuous analog of the result of [7], namely a classification of all discrete bispectral operators of the form $a(n) T+b(n)+c(n) T^{-1}$ (referred to as the extended Bochner-Krall problem in [14]). In [12] the Darboux process was applied to a biinfinite extension of the Laguerre difference operators considered in [10]. A large class of bispectral difference operators of the form above was thus constructed and many properties of the resulting objects were analyzed in detail. It is fair to say that the results in [12] provide a general treatment of the Laguerre case. The case of Jacobi difference operators has so far not been amenable to a similar treatment and only some special cases of Darboux maps were proved to preserve the bispectral property. The goal of the present paper is to provide such a general treatment in the Jacobi case and to state a conjecture for the classification problem above.

The rest of the introduction describes our results.

We take as a starting point the following natural extensions of the Jacobi polynomials, constructed in [10]:

$$
p_{\varepsilon}^{\alpha, \beta}(n, z)=\frac{(\varepsilon+\alpha+1)_{n}}{(\varepsilon+1)_{n}} F(-(n+\varepsilon), n+\varepsilon+\alpha+\beta+1, \alpha+1,(1-z) / 2) .
$$

Here and later we use $F$ for Gauss' ${ }_{2} F_{1}$ hypergeometric function. For negative integer values of $\alpha$, see (2.17). They are no longer polynomials but are still eigenfunctions of a biinfinite difference operator $L_{\alpha, \beta ; \varepsilon}(n, T)$ of the form $a_{0}(n) T+b_{0}(n)+c_{0}(n) T^{-1}$ and a differential operator $B_{\alpha, \beta}\left(z, \partial_{z}\right)$ :

$$
\begin{aligned}
& L_{\alpha, \beta ; \varepsilon}(n, T) p_{\varepsilon}^{\alpha, \beta}(n, z)=z p_{\varepsilon}^{\alpha, \beta}(n, z), \\
& B_{\alpha, \beta}\left(z, \partial_{z}\right) p_{\varepsilon}^{\alpha, \beta}(n, z)=\lambda_{\varepsilon}(n) p_{\varepsilon}^{\alpha, \beta}(n, z) .
\end{aligned}
$$

The operator $L_{\alpha, \beta ; \varepsilon}(n, T)$ is obtained by the formal change of variables $n \mapsto$ $n+\varepsilon$ from the standard (difference) Jacobi operator and is explicitly defined 
in (2.9). The operator $B_{\alpha, \beta}\left(z, \partial_{z}\right)$ and the spectral function $\lambda_{\varepsilon}(n)$ are given in Equations (2.5) and (2.12).

The sets of difference operators that we consider are obtained by the following version of the Darboux map starting from the operators $L_{\alpha, \beta ; \varepsilon}(n, T)$. Let $P(n, T)$ be a regular difference operator whose kernel is preserved by $L_{\alpha, \beta ; \varepsilon}(n, T)$. Then there exists a (unique) difference operator $L(n, T)$ such that

$$
L(n, T) P(n, T)=P(n, T) L_{\alpha, \beta ; \varepsilon}(n, T)
$$

which we refer to as a Darboux transformation from $L_{\alpha, \beta ; \varepsilon}(n, T)$. The advantage of this version is that this $L(n, T)$ is necessarily of the same form as $L_{\alpha, \beta ; \varepsilon}(n, T)$, i.e., $L(n, T)=a(n) T+b(n)+c(n) T^{-1}$ for some functions $a(n), b(n), c(n), n \in \mathbb{Z}$.

If $q(x)$ denotes the characteristic polynomial of the endomorphism $L(n, T)$ acting on the finite dimensional space $\operatorname{Ker} P(n, T)$, then

$$
\operatorname{Ker} P(n, T) \subset \operatorname{Ker} q\left(L_{\alpha, \beta ; \varepsilon}(n, T)\right) .
$$

In view of this it is natural to parametrize the sets of operators $L(n, T)$ by the Grassmannians of special subspaces of $\operatorname{Ker} q\left(L_{\alpha, \beta ; \varepsilon}(n, T)\right)$ that can occur as $\operatorname{Ker} P(n, T)$. Denote the set of difference operators $L(n, T)$ corresponding to characteristic polynomial $q(x)=(x-1)^{k}(x+1)^{l}$ by

$$
\mathcal{D}_{\alpha, \beta ; \varepsilon}^{(k, l)}
$$

The operators in $\mathcal{D}_{\alpha, \beta ; \varepsilon}^{(k, l)}$ are the main objects of study in this paper. Their explicit form is given in Section 3.3. Restricting to $q(x)$ with roots at \pm 1 guarantees that $L(n, T)$ will have rational coefficients. This is an important feature of bispectral operators. See [7] in the differential case.

It is an easy consequence of (1.3) that the function

$$
\Psi(n, z)=P(n, T) p_{\varepsilon}^{\alpha, \beta}(n, z)
$$

is an eigenfunction of the operator $L(n, T)$, namely we have

$$
L(n, T) \Psi(n, z)=z \Psi(n, z) .
$$

Our main result is:

Theorem 1.1. The difference operators $L(n, T)$ from the sets $\mathcal{D}_{\alpha, \beta ; \varepsilon}^{(k, l)}$ are bispectral (or more precisely the functions $\Psi(n, z)$ (1.4) are eigenfunctions of differential operators in the variable $z)$ in the following cases:

1) $\alpha \in \mathbb{Z}$ and $k \leq|\alpha|, l=0$,

2) $\beta \in \mathbb{Z}$ and $l \leq|\beta|, k=0$,

3) $\alpha, \beta \in \mathbb{Z}$ and $k \leq|\alpha|, l \leq|\beta|$. 
When $\varepsilon=0$, and $k=1$ and (or) $l=1$ these results were established in $[19,27]$. All this work starts with the classical paper of H.L. Krall, [20].

The proof of Theorem 1.1 is based on a general result of Bakalov, Horozov and Yakimov [3] which guarantees that a Darboux transformation preserves the bispectral property under some conditions on the operator $P(n, T)$. We will soon see that its application to the present situation is highly nontrivial and requires, in particular, an intrinsic characterization of a space of difference operators.

We will need some notation from [3], see Section 4.1 for more details. Denote by $\mathcal{B}_{\alpha, \beta ; \varepsilon}$ the algebra of difference operators $S(n, T)$ with rational coefficients for which there exists a differential operator $G\left(z, \partial_{z}\right)$ (also having rational coefficients) satisfying

$$
S(n, T) p_{\varepsilon}^{\alpha, \beta}(n, z)=G\left(z, \partial_{z}\right) p_{\varepsilon}^{\alpha, \beta}(n, z) .
$$

All such operators $S\left(z, \partial_{z}\right)$ form a "dual" algebra $\mathcal{B}_{\alpha, \beta ; \varepsilon}^{\prime}$. The map

$$
b: \mathcal{B}_{\alpha, \beta ; \varepsilon} \rightarrow \mathcal{B}_{\alpha, \beta ; \varepsilon}^{\prime}, \quad b(R(n, T))=S\left(z, \partial_{z}\right)
$$

is an antiisomorphism of associative algebras. Let $\mathcal{K}_{\alpha, \beta ; \varepsilon}$ and $\mathcal{K}_{\alpha, \beta ; \varepsilon}^{\prime}$ be the subalgebras of $\mathcal{B}_{\alpha, \beta ; \varepsilon}$ and $\mathcal{B}_{\alpha, \beta ; \varepsilon}^{\prime}$ consisting of rational functions. Bispectrality of $p_{\varepsilon}^{\alpha, \beta}(n, z)$ is equivalent to $\mathcal{K}_{\alpha, \beta ; \varepsilon}$ and $\mathcal{K}_{\alpha, \beta ; \varepsilon}^{\prime}$ being both nontrivial. Finally we arrive at the most important object for our consideration, namely the space

$$
\mathcal{R}_{\alpha, \beta ; \varepsilon}=\left\{(\mu(n))^{-1} P_{0}(n, T) \mid \mu(n) \in \mathcal{K}_{\alpha, \beta ; \varepsilon}, P_{0}(n, T) \in \mathcal{B}_{\alpha, \beta ; \varepsilon},\right.
$$

and the operator $(\mu(n))^{-1} P_{0}(n, T)$ does not have poles at $\left.n \in \mathbb{Z}\right\}$.

According to Theorem 1.2 of $[3], \Psi(n, z)$ is an eigenfunction of a differential operator in the variable $z$, if

$$
P(n, T) \in \mathcal{R}_{\alpha, \beta ; \varepsilon} .
$$

Thus to prove Theorem 1.1 we need a good description of the space $\mathcal{K}_{\alpha, \beta ; \varepsilon}$ which can be used to check whether the operators $P(n, T)$ from (1.3) belong to $\mathcal{R}_{\alpha, \beta ; \varepsilon}$. This is the hardest step in our paper. Let $\Delta$ denote the algebra of abstract difference operators with rational coefficients of the form $\sum_{i=p}^{q} b_{i}(n) T^{i}$ with rational functions $b_{i}(n)$ (possibly having poles in $\mathbb{Z}$ ). The key point of our approach is to consider the involution $I$ of $\Delta$ acting on rational functions $h(n)$ by

$$
(I h)(n):=h(-(n+2 \varepsilon+\alpha+\beta+1))
$$

and on the shift operator $T$ by $I(T):=T^{-1}$. In Section 4.2 we prove that $\mathcal{R}_{\alpha, \beta ; \varepsilon}$ consists of those difference operators from $\Delta$ that do not have poles 
in $\mathbb{Z}$ and after conjugation with the function

$$
\phi(n)=\frac{(\varepsilon+\alpha+1)_{n}}{(\varepsilon+1)_{n}}
$$

become $I$-invariant.

The final step of the proof of Theorem 1.1 is to show that the hypothesis guarantees that the kernel of the operator $P(n, T)$ (defining $L(n, T)$ ) has a basis of functions $f(n)$ for which the ratio $f(n) / \phi(n)$ is an (almost) $I$-invariant rational function in $n$. This is done in Section 5.1. Finally Section 5.2 recapitulates the strategy of the proof of Theorem 1.1 for the special case of the set $\mathcal{D}_{\alpha, \beta, \varepsilon}^{(2,0)}$. The reader may find it useful to consult this section while reading the paper.

Let us also note that in the case of Laguerre polynomials the situation simplifies a lot due to a presense of a relation of the type (1.5) with a difference operator $S(n, T)$ of the form $s_{1}(n) T+s_{0}(n)$ and a first order differential operator $G\left(z, \partial_{z}\right)$ (see expressions $(2.3)$ and $(2.8)$ in [12]). It is not hard to show that as a consequence of this the analog of $\mathcal{R}_{\alpha, \beta ; \varepsilon}$ in that case is simply the space of difference operators with rational coefficients.

Comparing with the differential case [7], it is natural to conjecture that all second order regular bispectral difference operators (i.e., having support $[-1,1])$ are exhausted by the families of operators constructed in $[\mathbf{1 0}, \mathbf{1 2}$, 16] and in this article. The operators in [16] are obtained as Darboux transformations from the operators $L_{\alpha, \beta ; \varepsilon}(n, T)$ for half integer values of the parameters $\alpha, \beta$ and are the analogs of "KdV family" in the differential case $[7]$.

For later use we introduce some convenient notation. If $f(n): \mathbb{Z} \rightarrow \mathbb{C}$ is a nowhere vanishing function and $D_{1}(n, T), D_{2}(n, T)$ are difference operators we denote

$$
\begin{aligned}
\operatorname{Ad}_{f(n)} D_{1}(n, T) & :=f(n) D_{1}(n, T) f(n)^{-1}, \\
\operatorname{ad}_{D_{2}(n, T)} D_{1}(n, T) & :=D_{2}(n, T) D_{1}(n, T)-D_{1}(n, T) D_{2}(n, T) .
\end{aligned}
$$

\section{Biinfinite Jacobi operators.}

In the first part of this section we review some properties of the classical Jacobi polynomials $p_{n}^{\alpha, \beta}(z)$. The second one discusses certain functions $p_{\varepsilon}^{\alpha, \beta}(n, z)$ which are eigenfunctions of biinfinite analogs $L_{\alpha, \beta ; \varepsilon}(n, T)$ of the Jacobi difference operators. The third part describes Darboux maps between the operators $L_{\alpha, \beta ; \varepsilon}(n, T)$ with shifted indices $\alpha, \beta$.

2.1. Jacobi polynomials. The Jacobi polynomials are the orthogonal polynomials for the measure $(1-z)^{\alpha}(1+z)^{\beta} d z$ on the interval $[-1,1]$, 
$(\alpha, \beta>-1)$, normalized by

$$
p_{n}^{\alpha, \beta}(1)=2^{-n}\left(\begin{array}{c}
n+\alpha \\
n
\end{array}\right), n \in \mathbb{Z}_{\geq 0} .
$$

They are given by

$$
p_{n}^{\alpha, \beta}(z)=\left(\begin{array}{c}
n+\alpha \\
n
\end{array}\right) F(-n, n+\alpha+\beta+1 ; \alpha+1 ;(1-z) / 2)
$$

where $F(a, b ; c ; x)$ denotes the Gauss' hypergeometric function. The reader can consult [21, pp. 209-217] for other explicit formulas and a list of major relations for $p_{n}^{\alpha, \beta}(z)$. Let

$$
p^{\alpha, \beta}(n, z)= \begin{cases}p_{n}^{\alpha, \beta}(z), & \text { for } n \in \mathbb{Z}_{\geq 0} \\ 0, & \text { for } n \in \mathbb{Z}_{<0} .\end{cases}
$$

Now $p^{\alpha, \beta}(n, z)$ are functions of a discrete parameter $n$ and a continuous parameter $z$. They satisfy a three term recursion relation

$$
L_{\alpha, \beta}(n, T) p^{\alpha, \beta}(n, z)=z p^{\alpha, \beta}(n, z)
$$

where $L_{\alpha, \beta}(n, T)$ are the difference operators

$$
\begin{aligned}
L_{\alpha, \beta}(n, T)= & \frac{2(n+1)(n+\alpha+\beta+1)}{(2 n+\alpha+\beta+1)(2 n+\alpha+\beta+2)} T \\
& +\frac{\beta^{2}-\alpha^{2}}{(2 n+\alpha+\beta)(2 n+\alpha+\beta+2)} \\
& +\frac{2(n+\alpha)(n+\beta)}{(2 n+\alpha+\beta)(2 n+\alpha+\beta+1)} T^{-1}
\end{aligned}
$$

called Jacobi operators. In addition $p^{\alpha, \beta}(n, z)$ are eigenfunctions of the differential operators $B_{\alpha, \beta}\left(z, \partial_{z}\right)$ given by

$$
B_{\alpha, \beta}\left(z, \partial_{z}\right)=\left(z^{2}-1\right) \partial_{z}^{2}+(\alpha-\beta+(\alpha+\beta+2) z) \partial_{z}
$$

i.e.,

$$
B_{\alpha, \beta}\left(z, \partial_{z}\right) p^{\alpha, \beta}(n, z)=\lambda(n) p^{\alpha, \beta}(n, z)
$$

for

$$
\lambda(n)=n(n+\alpha+\beta+1) .
$$

In view of $(2.3)$ and $(2.6), p^{\alpha, \beta}(n, z)$ are discrete-continuous bispectral functions and $L_{\alpha, \beta}(n, T), B\left(z, \partial_{z}\right)$ bispectral difference (differential) operators. 
2.2. The functions $p_{\varepsilon}^{\alpha, \beta}(n, z)$. In a study of the relation between the so called "associated Jacobi polynomials" and the discrete-continuous bispectral problem Grünbaum and Haine, see $[\mathbf{1 0}, \mathbf{1 1}, \mathbf{1 3}]$, introduced the functions

$$
p_{\varepsilon}^{\alpha, \beta}(n, z)=\frac{(\varepsilon+\alpha+1)_{n}}{(\varepsilon+1)_{n}} F(-(n+\varepsilon), n+\varepsilon+\alpha+\beta+1 ; \alpha+1 ;(1-z) / 2)
$$

$(n \in \mathbb{Z}, z \in \mathbb{C},|z|<1)$ defined for those $\varepsilon, \alpha, \beta \in \mathbb{C}$ such that $\alpha \notin \mathbb{Z}_{<0}$, and $\varepsilon \notin \mathbb{Z}_{<0}, \varepsilon+\alpha \notin \mathbb{Z}_{\geq 0}$. We will see later that the first restriction can be lifted.

The functions $p_{\varepsilon}^{\alpha, \beta}(n, z)$ are no longer polynomials but satisfy relations, similar to the ones for $p^{\alpha, \beta}(n, z)$. In particular they are eigenfunctions of the following difference operators with support $[-1,1]$

$$
\begin{aligned}
L_{\alpha, \beta ; \varepsilon}(n, T)= & \frac{2(n+\varepsilon+1)(n+\varepsilon+\alpha+\beta+1)}{(2 n+2 \varepsilon+\alpha+\beta+1)(2 n+2 \varepsilon+\alpha+\beta+2)} T \\
& +\frac{\beta^{2}-\alpha^{2}}{(2 n+2 \varepsilon+\alpha+\beta)(2 n+2 \varepsilon+\alpha+\beta+2)}+ \\
& +\frac{2(n+\varepsilon+\alpha)(n+\varepsilon+\beta)}{(2 n+2 \varepsilon+\alpha+\beta)(2 n+2 \varepsilon+\alpha+\beta+1)} T^{-1}
\end{aligned}
$$

and of the differential operators $B_{\alpha, \beta}\left(z, \partial_{z}\right)$, Equation (2.5). The corresponding relations are

$$
\begin{aligned}
L_{\alpha, \beta ; \varepsilon}(n, T) p_{\varepsilon}^{\alpha, \beta}(n, z) & =z p_{\varepsilon}^{\alpha, \beta}(n, z), \\
B_{\alpha, \beta}\left(z, \partial_{z}\right) p_{\varepsilon}^{\alpha, \beta}(n, z) & =\lambda_{\varepsilon}(n) p_{\varepsilon}^{\alpha, \beta}(n, z),
\end{aligned}
$$

where

$$
\lambda_{\varepsilon}(n)=(n+\varepsilon)(n+\varepsilon+\alpha+\beta+1) .
$$

The difference operators $L_{\alpha, \beta ; \varepsilon}(n, T)$ will still be called Jacobi operators. Further we will only deal with the case when they are regular, i.e., when their coefficients of $T$ and $T^{-1}$ do not vanish for $n \in \mathbb{Z}$. This amounts to the conditions

$$
\varepsilon, \varepsilon+\alpha, \varepsilon+\beta, \varepsilon+\alpha+\beta, 2 \varepsilon+\alpha+\beta \notin \mathbb{Z} .
$$

It may be useful to stress here that these will eventually be the only restrictions on our parameters $\alpha, \beta, \varepsilon$.

The operators $L_{\alpha, \beta ; \varepsilon}(n, T)$ do satisfy certain "transformation properties". For instance the following relations hold

(2.14) $L_{-\alpha,-\beta, \varepsilon+\alpha+\beta}(n, T)=L_{\alpha, \beta ; \varepsilon}(n, T)$,

(2.15) $\operatorname{Ad}_{(-1)^{n}} L_{\beta, \alpha, \varepsilon}(n, T)=\operatorname{Ad}_{(-1)^{n}} L_{-\beta,-\alpha, \varepsilon+\alpha+\beta}(n, T)=-L_{\alpha, \beta ; \varepsilon}(n, T)$. 
It is tempting to use (2.14) to limit attention to the case $\alpha \geq 0$. However, this would eventually bring an undesirable degree of asymmetry in the treatment of the parameters $\alpha$ and $\beta$. For this reason we prefer to introduce the appropriate functions $p_{\varepsilon}^{\alpha, \beta}(n, z)$ for $\alpha \in \mathbb{Z}_{<0}($ and $\varepsilon, \varepsilon+\beta, \varepsilon+\alpha, \varepsilon+\alpha+\beta \notin \mathbb{Z}$ ) by using (2.8) and recalling, see $\left[\mathbf{2 1}\right.$, p. 38] that for $m \in \mathbb{Z}_{\geq 0}$

$$
\begin{aligned}
& \lim _{c \rightarrow-m} \frac{1}{\Gamma(c)} F(a, b ; c ; z) \\
& =\frac{(a)_{m+1}(b)_{m+1}}{(m+1) !} z^{m+1} F(a+m+1, b+m+1 ; m+2 ; z) .
\end{aligned}
$$

We see below that this leads to the following expression for $p_{\varepsilon}^{\alpha, \beta}(n, z)$ with $\alpha \in \mathbb{Z}_{<0}$ (as long as (2.13) is satisfied)

$$
\begin{aligned}
\mathcal{C} & \frac{(\varepsilon+\beta+1)_{n}}{(\varepsilon+\alpha+\beta+1)_{n}} \\
& \quad \frac{(1-z)^{-\alpha}}{2^{-\alpha}} F(-(n+\varepsilon+\alpha), n+\varepsilon+\beta+1 ;-\alpha+1 ;(1-z) / 2)
\end{aligned}
$$

where the constant $\mathcal{C}=\mathcal{C}(\alpha, \beta, \varepsilon)$ is explicitly given by

$$
\mathcal{C}=\mathcal{C}(\alpha, \beta, \varepsilon)=\frac{(-1)^{\alpha}}{(-\alpha-1) !} \cdot \frac{(-\varepsilon)_{-\alpha}(\varepsilon+\alpha+\beta+1)_{-\alpha}}{(-\alpha) !} .
$$

It is easy to check that the assumptions (2.13) imply that $\mathcal{C}(\alpha, \beta, \varepsilon)$ is welldefined and does not vanish.

The expression above can be derived by a continuity argument using (2.8) and (2.16) when $\alpha$ approaches a value in $\mathbb{Z}_{<0}$. To see this it is important to notice that for $\alpha \in \mathbb{Z}_{<0}$ the identities

$$
\begin{aligned}
\frac{(\varepsilon+\alpha+1)_{n}}{(\varepsilon+1)_{n}} & =\frac{(-\varepsilon)_{-\alpha}}{(-(n+\varepsilon))_{-\alpha}} \quad \text { and } \\
\frac{(\varepsilon+\beta+1)_{n}}{(\varepsilon+\alpha+\beta+1)_{n}} & =\frac{(n+\varepsilon+\alpha+\beta+1)_{-\alpha}}{(\varepsilon+\alpha+\beta+1)_{-\alpha}}
\end{aligned}
$$

allow one to rewrite the factor

$$
\frac{(\varepsilon+\alpha+1)_{n}}{(\varepsilon+1)_{n}} \cdot \frac{(-(n+\varepsilon))_{-\alpha}(n+\varepsilon+\alpha+\beta+1)_{-\alpha}}{(-\alpha) !}
$$

as

$$
\frac{(-\alpha-1) !}{(-1)^{\alpha}} \cdot \mathcal{C}(\alpha, \beta, \varepsilon) \cdot \frac{(\varepsilon+\beta+1)_{n}}{(\varepsilon+\alpha+\beta+1)_{n}}
$$

which except for the first constant is the factor in front of (2.17).

Then conditions (2.13) guarantee that $p_{\varepsilon}^{\alpha, \beta}(n, z)$ is well-defined (see (2.8) and (2.17)) and satisfies (2.10) and (2.11). It was proved in [10] that the 
space of common solutions of (2.10) and (2.11) in a domain $\Omega \subset \mathbb{C}$, not containing \pm 1 , is two dimensional. Notice also that (2.13) excludes, in particular, the original operators $L_{\alpha, \beta}(n, z)(\varepsilon=0)$ since their leading coefficient vanishes for $n=-1$.

Finally we explain how (2.10) follows from (2.3). Conjugate the operator $L_{\alpha, \beta}(n, T)$ with $\left(\begin{array}{c}n+a \\ n\end{array}\right)=\frac{(\alpha+1)_{n}}{n !}$. The resulting difference operator has rational coefficients and the eigenfunction $F(-n, n+\alpha+\beta+1 ; \alpha+1 ;(1-z) / 2)$, cf. (2.1). The operator obtained from it by the formal change $n \mapsto n+\varepsilon$ has the eigenfunction $F(-n-\varepsilon, n+\varepsilon+\alpha+\beta+1 ; \alpha+1 ;(1-z) / 2)$ and all we need to do is conjugate it with $(\varepsilon+1)_{n} /(\varepsilon+\alpha+1)_{n}$. The result is the operator $L_{\alpha, \beta ; \varepsilon}(n, T)$ which proves $(2.10)$ in the case $\alpha \notin \mathbb{Z}_{<0}$. The case $\alpha \in \mathbb{Z}_{<0}$ follows from the definition (2.8) using the limit (2.16).

2.3. Darboux maps between Jacobi operators. There are four difference relations connecting the values of the Jacobi polynomials $p^{\alpha, \beta}(n, z)$ with shifted indices:

$$
\begin{aligned}
& p^{\alpha-1, \beta}(n, z)=\left(\frac{n+\alpha+\beta}{2 n+\alpha+\beta}-\frac{n+\beta}{2 n+\alpha+\beta} T^{-1}\right) p^{\alpha, \beta}(n, z), \\
& p^{\alpha, \beta}(n, z)=\frac{1}{z-1}\left(\frac{2(n+1)}{2 n+\alpha+\beta+1} T-\frac{2(n+\alpha)}{2 n+\alpha+\beta+1}\right) p^{\alpha-1, \beta}(n, z),
\end{aligned}
$$

and

$$
\begin{aligned}
& p^{\alpha, \beta-1}(n, z)=\left(\frac{n+\alpha+\beta}{2 n+\alpha+\beta}+\frac{n+\alpha}{2 n+\alpha+\beta} T^{-1}\right) p^{\alpha, \beta}(n, z), \\
& p^{\alpha, \beta}(n, z)=\frac{1}{z+1}\left(\frac{2(n+1)}{2 n+\alpha+\beta+1} T+\frac{2(n+\beta)}{2 n+\alpha+\beta+1}\right) p^{\alpha, \beta-1}(n, z),
\end{aligned}
$$

(see for instance, [21, Eqs. pp. 209-219]). Similarly to the proof of (2.10) at the end of the previous subsection, one shows the following analogs of these identities for $p_{\varepsilon}^{\alpha, \beta}(n, z)$

$p_{\varepsilon}^{\alpha-1, \beta}(n, z)=D_{-}^{\alpha}(n, T) p_{\varepsilon}^{\alpha, \beta}(n, z), p_{\varepsilon}^{\alpha+1, \beta}(n, z)=\frac{1}{z-1} D_{+}^{\alpha}(n, T) p_{\varepsilon}^{\alpha, \beta}(n, z)$,

$p_{\varepsilon}^{\alpha, \beta-1}(n, z)=D_{-}^{\beta}(n, T) p_{\varepsilon}^{\alpha, \beta}(n, z), p_{\varepsilon}^{\alpha, \beta+1}(n, z)=\frac{1}{z+1} D_{+}^{\beta}(n, T) p_{\varepsilon}^{\alpha, \beta}(n, z)$, 
where the operators $D_{ \pm}^{\alpha}(n, T)$ and $D_{ \pm}^{\beta}(n, T)$ are given by

$$
\begin{aligned}
& D_{-}^{\alpha}(n, T)=\left(\frac{\varepsilon+\alpha}{\alpha}\right)\left(\frac{n+\varepsilon+\alpha+\beta}{2 n+2 \varepsilon+\alpha+\beta}-\frac{n+\varepsilon+\beta}{2 n+2 \varepsilon+\alpha+\beta} T^{-1}\right), \\
& D_{+}^{\alpha}(n, T)=\left(\frac{\alpha+1}{\varepsilon+\alpha+1}\right)\left(\frac{2(n+\varepsilon+1)}{2 n+2 \varepsilon+\alpha+\beta+2} T-\frac{2(n+\varepsilon+\alpha+1)}{2 n+2 \varepsilon+\alpha+\beta+2}\right), \\
& D_{-}^{\beta}(n, T)=\left(\frac{n+\varepsilon+\alpha+\beta}{2 n+2 \varepsilon+\alpha+\beta}+\frac{n+\varepsilon+\alpha}{2 n+2 \varepsilon+\alpha+\beta} T^{-1}\right), \\
& D_{+}^{\beta}(n, T)=\left(\frac{2(n+\varepsilon+1)}{2 n+2 \varepsilon+\alpha+\beta+2} T+\frac{2(n+\varepsilon+\beta+1)}{2 n+2 \varepsilon+\alpha+\beta+2}\right) .
\end{aligned}
$$

The constant in (2.17) was chosen to make the relations (2.19)-(2.20) hold for all $\alpha \in \mathbb{C}$. We show only the dependence on the index $\alpha$ of the operators $D_{ \pm}^{\alpha}(n, T)$ because the index $\beta$ is unchanged in both sides of Equation (2.19), similarly for the operators $D_{ \pm}^{\beta}(n, T)$. Equations (2.19)-(2.20) and (2.10) imply the following factorizations

$$
\begin{aligned}
& L_{\alpha, \beta ; \varepsilon}(n, T)-1=D_{+}^{\alpha-1}(n, T) D_{-}^{\alpha}(n, T)=D_{-}^{\alpha+1}(n, T) D_{+}^{\alpha}(n, T), \\
& L_{\alpha, \beta ; \varepsilon}(n, T)+1=D_{+}^{\beta-1}(n, T) D_{-}^{\beta}(n, T)=D_{-}^{\beta+1}(n, T) D_{+}^{\beta}(n, T) .
\end{aligned}
$$

Hence the operators $L_{\alpha \pm 1, \beta ; \varepsilon}(n, T), L_{\alpha, \beta \pm 1 ; \varepsilon}(n, T)$ are Darboux transformations from $L_{\alpha, \beta ; \varepsilon}(n, T)$ and Equations (2.19) and (2.20) represent the Darboux maps $p_{\varepsilon}^{\alpha, \beta}(n, z) \mapsto p_{\varepsilon}^{\alpha \mp 1, \beta}(n, z)$ and $p_{\varepsilon}^{\alpha, \beta}(n, z) \mapsto p_{\varepsilon}^{\alpha, \beta \mp 1}(n, z)$.

\section{Darboux transformations from Jacobi operators.}

The first part of this section contains some general facts about discrete Darboux transformations in the form in which they will be used later (see, for instance, $[\mathbf{2 6}]$ for the differential case). The goal of the second part is an explicit description of the kernels of the operators $\left(L_{\alpha, \beta ; \varepsilon}-1\right)^{k}\left(L_{\alpha, \beta ; \varepsilon}+1\right)^{l}$. Based on it, in the third part we construct Darboux transformations from $L_{\alpha, \beta ; \varepsilon}(n, T)$ which are the main objects of study in the rest of the paper. The conditions (2.13) are assumed throughout Sections 3.2-3.3.

3.1. General remarks on Darboux transformations. One says that the difference operator $L(n, T)$ is obtained by a Darboux transformation from the difference operator $L_{0}(n, T)$ if there exists an operator $P(n, T)$ such that

$$
L(n, T) P(n, T)=P(n, T) L_{0}(n, T) .
$$

Assume that $L_{0}(n, T)$ has an eigenfunction $\Psi_{0}(n, z)$, i.e.,

$$
L_{0}(n, T) \Psi_{0}(n, T)=g_{0}(z) \Psi_{0}(n)
$$


for some function $g_{0}(z)$. Then

$$
\Psi(n, z):=P(n, T) \Psi_{0}(n, z)
$$

is an eigenfunction of $L(n, T)$ :

$$
L(n, T) \Psi(n, T)=g_{0}(z) \Psi(n) .
$$

The map $\Psi_{0}(n, T) \mapsto \Psi(n, T)$ is also called a Darboux transformation.

An important feature of the transformation (3.1) for a regular difference operator $P(n, T)$ is that the operator $L(n, T)$ has the same support as $L_{0}(n, T)$. Besides this $L(n, T)$ is regular if and only if $L_{0}(n, T)$ is regular.

Given a difference operator $L_{0}(n, T)$, all transformations of the type (3.1) with a regular difference operator $P(n, T)$ can be described in terms of the kernel of $P(n, T)$.

\section{Proposition 3.1.}

(i) For a regular difference operator $P(n, T)$ there exists an operator $L(n, T)$ for which (3.1) holds if and only if

$$
L_{0}(n, T)(\operatorname{Ker} P(n, T)) \subset \operatorname{Ker} P(n, T) .
$$

The operator $L(n, T)$ satisfying (3.1) is unique.

(ii) Let $P(n, T)$ be a regular difference operator satisfying (3.4) and $q(x)$ be the characteristic polynomial of the linear map $L_{0}(n, T)$ acting in the space $\operatorname{Ker} P(n, T)$. Then $\operatorname{Ker} P(n, T) \subset q\left(L_{0}(n, T)\right)$ and there exists an operator $Q(n, T)$ such that

$$
\begin{aligned}
& q\left(L_{0}(n, T)\right)=Q(n, T) P(n, T), \\
& q(L(n, T))=P(n, T) Q(n, T) .
\end{aligned}
$$

Note that the kernel of a regular difference operator $P(n, T)$ is finite dimensional. More precisely, if $P(n, T)$ has support $\left[m_{1}, m_{2}\right]$ for some $m_{i} \in$ $\mathbb{Z}$, then $\operatorname{dim} \operatorname{Ker} P(n, T)=m_{2}-m_{1}$. For any $j \in \mathbb{Z}$ the map

$$
f \mapsto\left(f(j+1), \ldots, f\left(j+m_{2}-m_{1}\right)\right), \text { for } f: \mathbb{Z} \rightarrow \mathbb{C}
$$

provides an isomorphism between $\operatorname{Ker} P(n, T)$ and $\mathbb{C}^{m_{2}-m_{1}}$.

The transformation $Q(n, T) P(n, T) \mapsto P(n, T) Q(n, T)$ is a more traditional version of the Darboux map. Although it is a special case of the transformation $L_{0}(n, T) \mapsto L(n, T)$ from Equation (3.1) and Proposition 3.1 shows that there always exists a polynomial $q(x)$ for which $q\left(L_{0}(n, T)\right) \mapsto$ $q(L(n, T))$ is a Darboux map in this sense.

Proof of Proposition 3.1. (i) If $P(n, T), L(n, T)$ satisfy (3.1) and $f(n) \in$ $\operatorname{Ker} P(n, T)$ then

$$
P(n, T)\left(L_{0}(n, T) f(n)\right)=L(n, T) P(n, T) f(n)=0
$$

which proves (3.4). 
In the opposite direction, let us notice that a comparison of the coefficients of the two sides of Equation (3.1) for a fixed value of $n$ gives a finite system for the corresponding coefficients of the unknown operator $L(n, T)$ having the same support as $L_{0}(n, T)$. One shows that it has a solution using the standard linear algebra fact that for a finite matrix $A$ the system $A u=b$ has a solution if and only if $v^{t} b=0, \forall v \in \operatorname{Ker} A^{t}$. In the particular case which we consider the last condition is fulfilled because of (3.4).

The regularity of the difference operator $P(n, T)$ implies the uniqueness of the operator $L(n, T)$ satisfying (3.1). Indeed if there are two such operators $L(n, T)$ and $L^{\prime}(n, T)$ one can subtract the resulting equalities (3.1). This gives $\left(L(n, T)-L^{\prime}(n, T)\right) P(n, T)=0$ which is a contradiction.

(ii) The relation $\operatorname{Ker} P(n, T) \subset q\left(L_{0}(n, T)\right)$ follows from the definition of $q(x)$. Similarly to Part (i), this implies the existence of an operator $Q(n, T)$ satisfying (3.5). Equations (3.1) and (3.5) imply

$$
q(L(n, T)) P(n, T)=P(n, T) q\left(L_{0}(n, T)\right)=(P(n, T) Q(n, T)) P(n, T)
$$

and as a consequence of this (3.6).

A regular difference operator is reconstructed from its kernel by the following lemma.

Lemma 3.2. Assume that $P(n, T)$ is a regular difference operator with support $\left[m_{1}, m_{2}\right]$ and leading coefficient 1 . Let $\operatorname{Ker} P(n, T)=\operatorname{Span}\left\{f^{(i)}(n)\right\}_{i=1}^{m}$ where $m=m_{2}-m_{2}$. Then the function

$$
\operatorname{det}(n):=\operatorname{det}\left(f^{(i)}(n-j)\right)_{i, j=1, m_{1}}^{m, m_{2}-1}
$$

does not vanish for $n \in \mathbb{Z}$ and

$$
P(n, T)=\frac{1}{\operatorname{det}(n)}\left|\begin{array}{cccc}
f^{(1)}\left(n+m_{1}\right) & \cdots & f^{(m)}\left(n+m_{1}\right) & T^{m_{1}} \\
\cdots & \cdots & \cdots & \cdots \\
f^{(1)}\left(n+m_{2}\right) & \cdots & f^{(m)}\left(n+m_{2}\right) & T^{m_{2}}
\end{array}\right|
$$

where the determinant is expanded from left to right (the shift operator $T$ does not commute with function multiplication).

Proof. The fact that the map (3.7) is an isomorphism between $\operatorname{Ker} P(n, T)$ and $\mathbb{C}^{m}$ implies that $\operatorname{det}(n)$ does not vanish for $n \in \mathbb{Z}$. Clearly the functions $f^{(i)}(n)$ belong to the kernel of the operator in the r.h.s. of (3.8). It has leading term 1 and the nonvanishing of $\operatorname{det}(n)$ implies (3.8).

Remark 3.3. The composition of two Darboux transformations $L_{0}(n, T)$ $\mapsto L_{1}(n, T)$ and $L_{1}(n, T) \mapsto L_{2}(n, T)$ of the type (3.1) is Darboux transformation $L_{0}(n, T) \mapsto L_{2}(n, T)$ of the same type. Indeed if

$$
L_{i}(n, T) P_{i}(n, T)=P_{i}(n, T) L_{i-1}(n, T), i=1,2,
$$

then

$$
L_{2}(n, T) P_{2}(n, T) P_{1}(n, T)=P_{2}(n, T) P_{1}(n, T) L_{0}(n, T) .
$$


3.2. Description of $\operatorname{Ker}\left(L_{\alpha, \beta ; \varepsilon}-1\right)^{k}\left(L_{\alpha, \beta ; \varepsilon}+1\right)^{l}$. The main idea is to first find some functions $\varphi(n, z)$ (depending on $\alpha, \beta$, and $\varepsilon$ ) such that

$$
L_{\alpha, \beta ; \varepsilon}(n, T) \varphi(n, z)=z \varphi(n, z)
$$

and then to consider the derivatives

$$
\varphi_{ \pm}^{(i)}(n)=\left.\frac{1}{i !} \partial_{z}^{i} \varphi(n, z)\right|_{z= \pm 1}, i \in \mathbb{Z}_{\geq 0}
$$

They satisfy

$$
\left(L_{\alpha, \beta ; \varepsilon}(n, T) \mp 1\right) \varphi_{ \pm}^{(i)}(n)=\varphi_{ \pm}^{(i-1)}(n), \forall i \in \mathbb{Z}_{\geq 0}
$$

with $\varphi_{ \pm}^{(-1)}(n)=0$. As a consequence of this

$$
\left(L_{\alpha, \beta ; \varepsilon}(n, T) \mp 1\right)^{i} \varphi_{ \pm}^{(j)}(n)=0, \forall i \in \mathbb{Z}_{>0}, j=0, \ldots, i-1 .
$$

Before stating the results from this subsection we recall a relation for the hypergeometric function that is a consequence of Gauss' relations between contiguous hypergeometric functions. Denote $F=F(a, b ; c ;(1-z) / 2), T F=$ $F(a-1, b+1 ; c ;(1-z) / 2)$, and $T^{-1} F=F(a+1, b-1 ; c ;(1-z) / 2)$. Then for $c \notin \mathbb{Z}_{\leq 0}$

$$
\begin{aligned}
& \frac{2(c-a) b}{(b-a)(b-a+1)} T F+\frac{2(a+b-1)(-2 c+a+b+1)}{(b-a-1)(b-a+1)} F \\
& +\frac{2 a(c-b)}{(b-a)(b-a-1)} T^{-1} F=z F
\end{aligned}
$$

This can also be checked directly using the standard expansion of $F(a, b ; c, x)$ for $|x|<1, c \notin \mathbb{Z}_{\leq 0}$

$$
F(a, b ; c ; x)=\sum_{j=0}^{\infty} \frac{(a)_{j}(b)_{j}}{j !(c)_{j}} x^{j} .
$$

Lemma 3.4. The four functions

$$
\begin{gathered}
\varphi_{+}(n, z)=\frac{(\varepsilon+\alpha+1)_{n}}{(\varepsilon+1)_{n}} \\
\cdot F(-(n+\varepsilon), n+\varepsilon+\alpha+\beta+1 ; \alpha+1 ;(1-z) / 2), \\
\psi_{+}(n, z)=\frac{(\varepsilon+\beta+1)_{n}}{(\varepsilon+\alpha+\beta+1)_{n}} \\
\cdot F(-(n+\varepsilon+\alpha+\beta), n+\varepsilon+1 ;-\alpha+1 ;(1-z) / 2),
\end{gathered}
$$




$$
\begin{aligned}
& \varphi_{-}(n, z)=\frac{(-1)^{n}(\varepsilon+\beta+1)_{n}}{(\varepsilon+1)_{n}} \\
& \cdot F(-(n+\varepsilon), n+\varepsilon+\alpha+\beta+1 ; \beta+1 ;(1+z) / 2), \\
& \psi_{-}(n, z)=\frac{(-1)^{n}(\varepsilon+\alpha+1)_{n}}{(\varepsilon+\alpha+\beta+1)_{n}} \\
& \quad \cdot F(-(n+\varepsilon+\alpha+\beta), n+\varepsilon+1 ;-\beta+1 ;(1+z) / 2)
\end{aligned}
$$

satisfy

$$
\left(L_{\alpha, \beta ; \varepsilon}(n, T)-z\right) \varphi_{ \pm}(n, z)=\left(L_{\alpha, \beta ; \varepsilon}(n, T)-z\right) \psi_{ \pm}(n, z)=0,
$$

provided that $\alpha \notin \mathbb{Z}_{<0}\left(\mathbb{Z}_{>0}\right)$ for $\varphi_{+}(n, z)\left(\psi_{+}(n, z)\right)$ and $\beta \notin \mathbb{Z}_{<0}\left(\mathbb{Z}_{>0}\right)$ for $\varphi_{-}(n, z)\left(\psi_{-}(n, z)\right)$.

Note that the assumptions (2.13) guarantee that the denominators of the first factors of $\varphi_{ \pm}(n, z)$ and $\psi_{ \pm}(n, z)$ do not vanish.

Proof. The relation (3.17) for $\varphi_{+}(n, z)$ holds because $\varphi_{+}(n, z)=p_{\varepsilon}^{\alpha, \beta}(n, z)$. To check the one for $\psi_{+}(n, z)$, we conjugate $L_{\alpha, \beta ; \varepsilon}(n, T)$ by $(\varepsilon+\beta+1)_{n} /(\varepsilon+$ $\alpha+\beta+1)_{n}$ (the factor in front of the r.h.s. of $(3.14)$ ).

The result is

$$
\begin{aligned}
& \operatorname{Ad}_{(\varepsilon+\beta+1)_{n} /(\varepsilon+\alpha+\beta+1)_{n} L_{\alpha, \beta ; \varepsilon}(n, T)} \\
& =\frac{2(n+\varepsilon+1)(n+\varepsilon+\beta+1)}{(2 n+2 \varepsilon+\alpha+\beta+1)(2 n+2 \varepsilon+\alpha+\beta+2)} T \\
& \quad+\frac{\beta^{2}-\alpha^{2}}{(2 n+2 \varepsilon+\alpha+\beta)(2 n+2 \varepsilon+\alpha+\beta+2)} \\
& \quad+\frac{2(n+\varepsilon+\alpha)(n+\varepsilon+\alpha+\beta)}{(2 n+2 \varepsilon+\alpha+\beta)(2 n+2 \varepsilon+\alpha+\beta+1)} T^{-1} .
\end{aligned}
$$

This is the difference operator from the l.h.s. of (3.11) with $a=-(n+$ $\varepsilon+\alpha+\beta), b=n+\varepsilon+1$, and $c=-\alpha+1$ which gives the proof of (3.17) for $\psi_{+}(n, z)$. The cases of $\varphi_{-}(n, z)$ and $\psi_{-}(n, z)$ are handled in a similar fashion.

Next we consider the derivatives of $\varphi_{+}(n, z), \psi_{+}(n, z)$ at $z=1$ and of $\varphi_{-}(n, z), \psi_{-}(n, z)$ at $z=-1$ :

$$
\begin{aligned}
& \varphi_{ \pm}^{(i)}(n):=\left.\frac{1}{i !} \partial_{z}^{i} \varphi_{ \pm}(n, z)\right|_{z= \pm 1} \\
& \psi_{ \pm}^{(i)}(n):=\left.\frac{1}{i !} \partial_{z}^{i} \psi_{ \pm}(n, z)\right|_{z= \pm 1}
\end{aligned}
$$

$i \in \mathbb{Z}_{\geq 0}$ (with the restrictions on $\alpha$ and $\beta$ made at the end of Lemma 3.4). 
Using the expansion (3.12) of the hypergeometric function, we obtain the following explicit formulas for $\varphi_{ \pm}^{(i)}(n)$ and $\psi_{ \pm}^{(i)}(n)$

$$
\begin{aligned}
& \varphi_{+}^{(i)}(n)=\frac{(\varepsilon+\alpha+1)_{n}}{(\varepsilon+1)_{n}} \cdot \frac{(-(n+\varepsilon))_{i}(n+\varepsilon+\alpha+\beta+1)_{i}}{(-2)^{i} i !(\alpha+1)_{i}} \\
& \psi_{+}^{(i)}(n)=\frac{(\varepsilon+\beta+1)_{n}}{(\varepsilon+\alpha+\beta+1)_{n}} \cdot \frac{(-(n+\varepsilon+\alpha+\beta))_{i}(n+\varepsilon+1)_{i}}{(-2)^{i} i !(-\alpha+1)_{i}} \\
& \varphi_{-}^{(i)}(n)=\frac{(-1)^{n}(\varepsilon+\beta+1)_{n}}{(\varepsilon+1)_{n}} \cdot \frac{(-(n+\varepsilon))_{i}(n+\varepsilon+\alpha+\beta+1)_{i}}{2^{i} i !(\beta+1)_{i}} \\
& \psi_{-}^{(i)}(n)=\frac{(-1)^{n}(\varepsilon+\alpha+1)_{n}}{(\varepsilon+\alpha+\beta+1)_{n}} \cdot \frac{(-(n+\varepsilon+\alpha+\beta))_{i}(n+\varepsilon+1)_{i}}{2^{i} i !(-\beta+1)_{i}}
\end{aligned}
$$

We define $\varphi_{+}^{(i)}(n)\left(\psi_{+}^{(i)}(n)\right)$ for $\alpha \in \mathbb{Z}_{<0}\left(\alpha \in \mathbb{Z}_{>0}\right), i<|\alpha|$ by (3.18), (3.19) and $\varphi_{-}^{(i)}(n)\left(\psi_{-}^{(i)}(n)\right)$ for $\beta \in \mathbb{Z}_{<0}\left(\beta \in \mathbb{Z}_{>0}\right), i<|\beta|$ by (3.20), (3.21). (Note that these cases were excluded in Lemma 3.4.)

Theorem 3.5. Assuming (2.13) the following relations

$$
\begin{aligned}
& \left(L_{\alpha, \beta ; \varepsilon}(n, T)-1\right) \varphi_{+}^{(i)}(n)=\varphi_{+}^{(i-1)}(n), \\
& \left(L_{\alpha, \beta ; \varepsilon}(n, T)-1\right) \psi_{+}^{(i)}(n)=\psi_{+}^{(i-1)}(n),
\end{aligned}
$$

hold for all $i \in \mathbb{Z}_{>0}$ if $\alpha \notin \mathbb{Z}$ and for $i=0, \ldots,|\alpha|-1$ if $\alpha \in \mathbb{Z}$. Similarly one has

$$
\begin{aligned}
& \left(L_{\alpha, \beta ; \varepsilon}(n, T)+1\right) \varphi_{-}^{(i)}(n)=\varphi_{-}^{(i-1)}(n), \\
& \left(L_{\alpha, \beta ; \varepsilon}(n, T)+1\right) \psi_{-}^{(i)}(n)=\psi_{-}^{(i-1)}(n),
\end{aligned}
$$

for all $i \in \mathbb{Z}_{>0}$ if $\beta \notin \mathbb{Z}$ and for $i=0, \ldots,|\beta|-1$ if $\beta \in \mathbb{Z}$. (We set $\varphi_{ \pm}^{(-1)}(n)=\psi_{ \pm}^{(-1)}(n)=0$.)

The kernels of $\left(L_{\alpha, \beta ; \varepsilon}(n, T)-1\right)^{k}$ and $\left(L_{\alpha, \beta ; \varepsilon}(n, T)+1\right)^{l}$ are given by

$$
\begin{aligned}
& \operatorname{Ker}\left(L_{\alpha, \beta ; \varepsilon}(n, T)-1\right)^{k}=\operatorname{Span}\left\{\varphi_{+}^{(i)}(n), \psi_{+}^{(i)}(n)\right\}_{i=0}^{k-1}, \\
& \operatorname{Ker}\left(L_{\alpha, \beta ; \varepsilon}(n, T)+1\right)^{l}=\operatorname{Span}\left\{\varphi_{-}^{(i)}(n), \psi_{-}^{(i)}(n)\right\}_{i=0}^{l-1},
\end{aligned}
$$

for $k \leq|\alpha|$ if $\alpha \in \mathbb{Z}$, for $l \leq|\beta|$ if $\beta \in \mathbb{Z}$, and for all $k, l \geq 0$ if $\alpha, \beta \notin \mathbb{Z}$.

Proof. In the case $\alpha \notin \mathbb{Z}$, the functions $\varphi_{+}(n, z)$ and $\psi_{+}(n, z)$ are welldefined. From the remark in the beginning of this subsection it follows that (3.17) and the definitions of $\varphi_{+}^{(j)}(n), \psi_{+}^{(j)}(n)$ imply (3.22), (3.23). The case $\alpha \in \mathbb{Z}, i<|\alpha|$ follows by continuity on $\alpha$.

The inclusion $\supset$ in (3.26), (3.27) clearly follows from (3.22)-(3.25). Because $\left(L_{\alpha, \beta ; \varepsilon}(n, T)-1\right)^{k}$ is a regular difference operator with support $[-k, k]$, to prove $(3.26)$ it suffices to show that the functions $\varphi_{+}^{(i)}(n), \psi_{+}^{(i)}(n), i=$ $0, \ldots, k-1$ are linearly independent. 
Assume that

$$
\sum_{i=0}^{k_{0}}\left(a_{i} \varphi_{+}^{(i)}(n)+b_{i} \psi_{+}^{(i)}(n)\right)=0, \quad \forall n \in \mathbb{Z}
$$

for some complex numbers $a_{0}, \ldots, a_{k_{0}}, b_{0}, \ldots, b_{k_{0}}$, such that $a_{k_{0}} \neq 0$ or $b_{k_{0}} \neq 0\left(k_{0} \leq k-1\right)$. Applying $\left(L_{\alpha, \beta ; \varepsilon}(n, T)\right)^{k_{0}-1}$ to this equality and using (3.22), (3.23), we get

$$
a_{k_{0}} \varphi_{+}^{(0)}(n)+b_{k_{0}} \psi_{+}^{(0)}(n)=0, \quad \forall n \in \mathbb{Z},
$$

i.e.,

$$
a_{k_{0}} \frac{(\varepsilon+\alpha+1)_{n}}{(\varepsilon+1)_{n}}=-b_{k_{0}} \frac{(\varepsilon+\beta+1)_{n}}{(\varepsilon+\alpha+\beta+1)_{n}}, \quad \forall n \in \mathbb{Z} .
$$

For $n=0$ this gives $a_{k_{0}}=-b_{k_{0}}(\neq 0)$. Dividing the two sides of Equation (3.28) for two consecutive values of $n$, we get

$$
(\varepsilon+\alpha+n)(\varepsilon+\alpha+\beta+n)=(\varepsilon+n)(\varepsilon+\beta+n), \quad \forall n \in \mathbb{Z} .
$$

This gives $\alpha=0$ which is a contradiction. Equation (3.27) is proved analogously.

Remark 3.6. It is clear that

$$
\operatorname{Ker}\left(L_{\alpha, \beta ; \varepsilon}-1\right)^{k} \cap \operatorname{Ker}\left(L_{\alpha, \beta ; \varepsilon}+1\right)^{l}=\emptyset .
$$

Therefore

$$
\operatorname{Ker}\left(L_{\alpha, \beta ; \varepsilon}-1\right)^{k}\left(L_{\alpha, \beta ; \varepsilon}+1\right)^{l}=\operatorname{Ker}\left(L_{\alpha, \beta ; \varepsilon}-1\right)^{k} \oplus \operatorname{Ker}\left(L_{\alpha, \beta ; \varepsilon}+1\right)^{l}
$$

and Theorem 3.5 describes the kernel of the operator $\left(L_{\alpha, \beta ; \varepsilon}-1\right)^{k}\left(L_{\alpha, \beta ; \varepsilon}+1\right)^{l}$ in the cases specified there.

3.3. The sets $\mathcal{D}_{\alpha, \beta ; \varepsilon}^{(k, l)}$ of Darboux transformations from $L_{\alpha, \beta ; \varepsilon}(n, z)$. Let us fix two nonnegative integers $k$ and $l$ and choose $2(k+l)$ complex numbers

$$
\begin{aligned}
& A_{i}, B_{i}, i=0, \ldots, k-1, \\
& C_{j}, D_{j}, j=0, \ldots, l-1 .
\end{aligned}
$$

If $k>0(l>0)$ we will assume $\alpha \neq-k+1, \ldots, k-1(\beta \neq-l+1, \ldots, l-1)$. Set

$$
\begin{aligned}
& f^{(i)}(n) \\
& =\left\{\begin{array}{ll}
\sum_{r=0}^{i}\left(A_{r} \varphi_{+}^{(i-r)}(n)+B_{r} \psi_{+}^{(i-r)}(n)\right), & \text { for } i=0, \ldots, k-1 \\
\sum_{r=0}^{i-k}\left(C_{r} \varphi_{-}^{(i-k-r)}(n)+D_{r} \psi_{-}^{(i-k-r)}(n)\right), & \text { for } i=k, \ldots, k+l-1
\end{array} .\right.
\end{aligned}
$$


The values of the parameters $A, B, C, D \in \mathbb{C}$ for which

$$
\operatorname{det}(n)=\operatorname{det}\left(f^{(i)}(n+j)\right)_{i, j=0,-k-l}^{k+l-1,-1} \neq 0, \forall n \in \mathbb{Z},
$$

will be called admissible. For such values we define the operator

$$
P(n, T)=\frac{1}{\operatorname{det}(n)}\left|\begin{array}{cccc}
f^{(0)}(n-k-l) & \ldots & f^{(k+l-1)}(n-k-l) & T^{-(k+l)} \\
\ldots & \ldots & \ldots & \ldots \\
f^{(0)}(n) & \ldots & f^{(k+l-1)}(n) & 1
\end{array}\right| .
$$

By expanding (3.30) along the last column one sees that the term of $T^{-(k+l)}$ is given by

$$
\frac{\operatorname{det}(n+1)}{\operatorname{det}(n)} \neq 0
$$

hence $P(n, T)$ is a regular difference operator. As a consequence of properties (3.22)-(3.25) we obtain

$$
\left(L_{\alpha, \beta ; \varepsilon}(n, T)-1\right) f^{(0)}(n)=0, \quad\left(L_{\alpha, \beta ; \varepsilon}(n, T)-1\right) f^{(i)}=f^{(i-1)}(n)
$$

for $i=1, \ldots, k-1$ and

$$
\left(L_{\alpha, \beta ; \varepsilon}(n, T)+1\right) f^{(k)}(n)=0, \quad\left(L_{\alpha, \beta ; \varepsilon}(n, T)+1\right) f^{(j)}=f^{(j-1)}(n)
$$

for $j=k+1, \ldots, k+l-1$. Thus $\operatorname{Ker} P(n, T)=\operatorname{Span}\left\{f^{(i)}(n)\right\}_{i=0}^{k+l-1}$ is preserved by $L_{\alpha, \beta ; \varepsilon}(n, T)$ and according to Proposition 3.1 there exists a difference operator $L(n, T)$ with support $[-1,1]$ such that

$$
L(n, T) P(n, T)=P(n, T) L_{\alpha, \beta ; \varepsilon}(n, T) .
$$

The set of all difference operators $L(n, T)$ for admissible values of the parameters $A, B, C, D$ will be denoted by

$$
\mathcal{D}_{\alpha, \beta ; \varepsilon}^{(k, l)} \text {. }
$$

All operators $L(n, T) \in \mathcal{D}_{\alpha, \beta ; \varepsilon}^{(k, l)}$ are Darboux transformations from $L_{\alpha, \beta ; \varepsilon}(n, T)$ and $k, l$ refer to the multiplicity of the eigenvalues 1 and -1 of $L_{\alpha, \beta ; \varepsilon}(n, T)$ in $\operatorname{Ker} P(n, T)$, see Equations (3.31) and (3.32). (Recall from part (i) of Proposition 3.1 that $L_{\alpha, \beta ; \varepsilon}(n, T)$ preserves $\operatorname{Ker} P(n, T)$.) Every $L(n, T) \in$ $\mathcal{D}_{\alpha, \beta ; \varepsilon}^{(k, l)}$ is a regular difference operator with eigenfunction

$$
\Psi(n, z)=P(n, T) p_{\varepsilon}^{\alpha, \beta}(n, z),
$$

more precisely:

$$
L(n, T) \Psi(n, z)=z \Psi(n, z) .
$$

The admissibility condition (3.29) holds for almost all values of $A, B, C, D$ $\in \mathbb{Z}$. The complement of the corresponding set in $\mathbb{C}^{2(k+l)}$ consists of the zeros of countably many polynomials, obtained from $\operatorname{det}(n)$ for fixed $n \in$ 
$\mathbb{Z}$ (recall (3.29)). The latter do not vanish identically due to the linear independence of the set of functions $\left\{\varphi_{ \pm}^{(i)}(n)\right\}_{i=0}^{k-1} \cup\left\{\psi_{ \pm}^{(j)}(n)\right\}_{j=0}^{l-1}$ (see the proof of Theorem 3.5) and the regularity of $L_{\alpha, \beta ; \varepsilon}(n, T)$.

There are in fact $k+l$ free parameters in the definition of an element $L(n, T) \in \mathcal{D}_{\alpha, \beta ; \varepsilon}^{(k, l)}$ since the operator $P(n, T)$ (see (3.30)) only depends on the choice of the space $\operatorname{Span}\left\{f^{(i)}(n)\right\}_{i=0}^{k+l-1}(=\operatorname{Ker} P(n, T))$, and not on the choice of the individual functions $f^{(i)}(n)$. Using again the linear independence of $\left\{\varphi_{ \pm}^{(i)}(n)\right\}_{i=0}^{k-1} \cup\left\{\psi_{ \pm}^{(j)}(n)\right\}_{j=0}^{l-1}$, the choice of span is equivalent to a choice of flags

$$
V_{0} \subset V_{1} \subset \ldots \subset V_{k-1} \text { and } W_{0} \subset W_{1} \subset \ldots \subset W_{l-1}
$$

where $V_{i}=\operatorname{Span}\left\{f^{(r)}(n)\right\}_{r=0}^{i}$ and $W_{j}=\operatorname{Span}\left\{f^{(r)}(n)\right\}_{r=k}^{k+j}$, cf. [12].

The relations (2.14) and (2.15) for $L_{\alpha, \beta ; \varepsilon}(n, T)$ imply similar relations for the sets $\mathcal{D}_{\alpha, \beta ; \varepsilon}^{(k, l)}$ :

$$
\begin{aligned}
& \mathcal{D}_{-\alpha,-\beta, \varepsilon+\alpha+\beta}^{(k, l)}=\mathcal{D}_{\alpha, \beta ; \varepsilon}^{(k, l)}, \\
& \operatorname{Ad}_{(-1)^{n}} \mathcal{D}_{\beta, \alpha, \varepsilon}^{(l, k)}=\operatorname{Ad}_{(-1)^{n}} \mathcal{D}_{-\beta,-\alpha, \varepsilon+\alpha+\beta}^{(l, k)}=-\mathcal{D}_{\alpha, \beta ; \varepsilon}^{(k, l)} .
\end{aligned}
$$

Here, in addition to (2.14) and (2.15), we use that the change of parameters $\alpha \rightarrow-\alpha, \beta \rightarrow-\beta, \varepsilon \rightarrow \varepsilon+\alpha+\beta$ exchanges $\varphi_{+}^{(i)}(n)$ with $\psi_{+}^{(i)}(n)$ and $\varphi_{-}^{(i)}(n)$ with $\psi_{-}^{(i)}(n)$. Analogously the change of parameters $\alpha \rightarrow \beta, \beta \rightarrow \alpha, \varepsilon \rightarrow \varepsilon$ exchanges $\varphi_{+}^{(i)}(n)$ with $(-1)^{n} \varphi_{-}^{(i)}(n)$ and $\psi_{+}^{(i)}(n)$ with $(-1)^{n} \psi_{-}^{(i)}(n)$.

The Darboux maps between Jacobi functions (operators) represented by the first identities in (2.19), (2.20) and Remark 3.3 imply the following inclusion relations

$$
\begin{aligned}
& \operatorname{Ad}_{\frac{2 n+2 \varepsilon+\alpha+\beta}{n+\varepsilon+\alpha+\beta}} \mathcal{D}_{\alpha-1, \beta ; \varepsilon}^{(k-1, l)} \subset \mathcal{D}_{\alpha, \beta ; \varepsilon}^{(k, l)}, \\
& \operatorname{Ad}_{\frac{2 n+2 \varepsilon+\alpha+\beta}{n+\varepsilon+\alpha+\beta}} \mathcal{D}_{\alpha, \beta-1 ; \varepsilon}^{(k, l-1)} \subset \mathcal{D}_{\alpha, \beta ; \varepsilon}^{(k, l)} .
\end{aligned}
$$

The function $(n+\varepsilon+\alpha+\beta) /(2 n+2 \varepsilon+\alpha+\beta)$ is the leading coefficient of the the operators $D_{-}^{\alpha}(n, T)$ and $D_{-}^{\beta}(n, T)$, see Section 2.3. Recall that the operator $P(n, T)$ is normalized to have leading coefficient 1 .

Remark 3.7. Note that (3.31), (3.32) imply that for the operator $P(n, T)$ (3.30) defining an element $L(n, T)$ in $\mathcal{D}_{\alpha, \beta ; \varepsilon}^{(k, l)}$ the endomorphism $L_{\alpha, \beta ; \varepsilon}(n, T)$ on $\operatorname{Ker} P(n, T)$ has two Jordan blocks with eigenvalues 1 and -1 and lengths $k$ and $l$, respectively. Insisting on multiple blocks with equal eigenvalues does not produce larger sets of transformations since the operator $L_{\alpha, \beta ; \varepsilon}(n, T)$ has a two dimensional kernel. Allowing $k>|\alpha|$ or $l>\beta$ in the cases $\alpha \in \mathbb{Z}$ or $\beta \in \mathbb{Z}$ causes the operators $P(n, T)$ and $L(n, T)$ to have nonrational coefficients which does lead to bispectrality of $L(n, T)$ as was noted in the introduction. 
At the end of this subsection we compute explicitly the coefficients of the operators $L(n, T)$ in $\mathcal{D}_{\alpha, \beta ; \varepsilon}^{(k, l)}$. Set

$$
L(n, T)=a(n) T+b(n)+c(n) T^{-1}
$$

for some functions $a(n), b(n)$, and $c(n)$ (the dependence on $A, B, C, D$ will not be shown). For convenience we denote the coefficients of the operator $L_{\alpha, \beta ; \varepsilon}(n, T)$ by $a_{0}(n), b_{0}(n)$, and $c_{0}(n)$ :

$$
L_{\alpha, \beta ; \varepsilon}(n, T)=a_{0}(n) T+b_{0}(n)+c_{0}(n) T^{-1}
$$

(cf. Equation (2.9) for their values). Set also

$$
\operatorname{det}_{-r}(n):=\operatorname{det}\left(f^{(i)}(n+j)\right)_{\substack{i=0, \ldots, k+l-1 \\ j=-k-l, \ldots,-\hat{r}, \ldots, 0}} \text { for } r=0, \ldots, k+l .
$$

Note that

$$
\operatorname{det}_{0}(n)=\operatorname{det}(n) \quad \text { and } \operatorname{det}_{k+l}(n)=\operatorname{det}_{0}(n+1)=\operatorname{det}(n+1) .
$$

Expanding the determinant (3.30) defining $P(n, T)$ along the last column gives

$$
P(n, T)=\sum_{r=0}^{k+l}(-1)^{r} \frac{\operatorname{det}_{-r}(n)}{\operatorname{det}(n)} T^{-r} .
$$

Proposition 3.8. The coefficients $a(n), b(n)$, and $c(n)$ of an operator $L(n, T) \in \mathcal{D}_{\alpha, \beta ; \varepsilon}^{(k, l)}$ are expressed in terms of the coefficients $a_{0}(n), b_{0}(n)$, and $c_{0}(n)$ of $L_{\alpha, \beta ; \varepsilon}(n, T)$ and the functions $f^{(i)}(n)$ (see (3.42)) by the following formulas

$$
\begin{aligned}
& a(n)=a_{0}(n), \\
& b(n)=b_{0}(n)+a_{0}(n) \frac{\operatorname{det}_{-1}(n+1)}{\operatorname{det}(n+1)}-a_{0}(n-1) \frac{\operatorname{det}_{-1}(n)}{\operatorname{det}(n)}, \\
& c(n)=c_{0}(n-k-l) \frac{\operatorname{det}(n-1) \operatorname{det}(n+1)}{(\operatorname{det}(n))^{2}} .
\end{aligned}
$$

Proof. We compare the coefficients of $T$ and 1 in (3.33) and use the expression (3.44) for the operator $P(n, T)$. This gives the formulas

$$
\begin{aligned}
& a(n)=a_{0}(n), \\
& b(n)-a(n) \frac{\operatorname{det}_{-1}(n+1)}{\operatorname{det}(n+1)}=b_{0}(n)-a_{0}(n-1) \frac{\operatorname{det}_{-1}(n)}{\operatorname{det}(n)},
\end{aligned}
$$

which are equivalent to (3.45) and (3.46).

Similarly comparing the coefficients of $T^{-k-l-1}$ in (3.33) gives

$$
c(n) \frac{\operatorname{det}_{-(k+l)}(n-1)}{\operatorname{det}(n-1)}=c_{0}(n-k-l) \frac{\operatorname{det}_{-(k+l)}(n)}{\operatorname{det}(n)}
$$

which implies (3.47), taking into account (3.43). 


\section{Bispectral Darboux transformation and an involution.}

This section is a preparation for the next one where we show that the difference operators from $\mathcal{D}_{\alpha, \beta ; \varepsilon}^{(k, l)}$ are bispectral under some natural conditions on $\alpha$ and $\beta$. Our proof is based on a result of [3] on Darboux transformations that preserve the bispectral property. Its application to the situation under consideration is nontrivial and requires an intrinsic characterization of a certain space of difference operators. This is done in terms of an involution of the algebra of difference operators with rational coefficients.

4.1. A theorem on bispectral Darboux transformations. For a fixed choice of the parameters $\alpha, \beta, \varepsilon$ we define $\mathcal{B}_{\alpha, \beta ; \varepsilon}$ as the algebra of difference operators $S(n, T)$ with rational coefficients for which there exists a differential operator $G\left(z, \partial_{z}\right)$ (also with rational coefficients) such that

$$
S(n, T) p_{\varepsilon}^{\alpha, \beta}(n, z)=G\left(z, \partial_{z}\right) p_{\varepsilon}^{\alpha, \beta}(n, z) .
$$

The set of all such operators $G\left(z, \partial_{z}\right)$ is an algebra which will be denoted by $\mathcal{B}_{\alpha, \beta ; \varepsilon}^{\prime}$. It is clear that

$$
b(S(n, T)):=G\left(z, \partial_{z}\right)
$$

correctly defines a map

$$
b: \mathcal{B}_{\alpha, \beta ; \varepsilon} \rightarrow \mathcal{B}_{\alpha, \beta ; \varepsilon}^{\prime}
$$

which is an antiisomorphism of algebras. In this setting Equations (2.10) and (2.11) mean that $\lambda_{\varepsilon}(n), L_{\alpha, \beta ; \varepsilon}(n, T) \in \mathcal{B}_{\alpha, \beta ; \varepsilon}, z, B_{\alpha, \beta}\left(z, \partial_{z}\right) \in \mathcal{B}_{\alpha, \beta ; \varepsilon}^{\prime}$, and

$$
\begin{aligned}
& b\left(\lambda_{\varepsilon}(n)\right)=B_{\alpha, \beta}\left(z, \partial_{z}\right), \\
& b\left(L_{\alpha, \beta ; \varepsilon}(n, T)\right)=z .
\end{aligned}
$$

The triple $\left(\mathcal{B}_{\alpha, \beta ; \varepsilon}, \mathcal{B}_{\alpha, \beta ; \varepsilon}^{\prime}, b\right)$ is an example of a bispectral triple in the sense of $[3]$. Denote

$$
\begin{aligned}
& \mathcal{K}_{\alpha, \beta ; \varepsilon}=\mathcal{B}_{\alpha, \beta ; \varepsilon} \cap \mathbb{C}(n), \\
& \mathcal{K}_{\alpha, \beta ; \varepsilon}^{\prime}=\mathcal{B}_{\alpha, \beta ; \varepsilon}^{\prime} \cap \mathbb{C}(z),
\end{aligned}
$$

where $\mathbb{C}(n)$ and $\mathbb{C}(z)$ stand for the algebras of rational functions in the variables $n$ and $z$, respectively. Let

$$
\begin{aligned}
& \mathcal{A}_{\alpha, \beta ; \varepsilon}=b^{-1}\left(\mathcal{K}_{\alpha, \beta ; \varepsilon}^{\prime}\right), \\
& \mathcal{A}_{\alpha, \beta ; \varepsilon}^{\prime}=b\left(\mathcal{K}_{\alpha, \beta ; \varepsilon}\right) .
\end{aligned}
$$

It is obvious that

$$
\begin{aligned}
& \mathcal{K}_{\alpha, \beta ; \varepsilon}^{\prime}=\mathbb{C}[z], \\
& \mathcal{A}_{\alpha, \beta ; \varepsilon}^{\prime}=\mathbb{C}\left[B_{\alpha, \beta}\left(z, \partial_{z}\right)\right],
\end{aligned}
$$


and

$$
\begin{aligned}
& \mathcal{K}_{\alpha, \beta ; \varepsilon} \supset \mathbb{C}[\lambda(n)], \\
& \mathcal{A}_{\alpha, \beta ; \varepsilon} \supset \mathbb{C}\left[L_{\alpha, \beta ; \varepsilon}(n, T)\right] .
\end{aligned}
$$

Later in Remark 4.3 we will show that the inclusions in (4.12) and (4.13) can be strengthen to give two equalities.

As was noted in Section 3.1, if a difference operator $q\left(L_{\alpha, \beta ; \varepsilon}(n, T)\right) \in$ $\mathcal{A}_{\alpha, \beta ; \varepsilon}(q(x) \in \mathbb{C}[x])$ is factorized as a product of two operators $Q(n, T)$ and $P(n, T)$

$$
q\left(L_{\alpha, \beta ; \varepsilon}(n, T)\right)=Q(n, T) P(n, T),
$$

then the function

$$
\Psi(n, z)=P(n, T) p_{\varepsilon}^{\alpha, \beta}(n, z)
$$

is an eigenfunction of the difference operator $P(n, T) Q(n, T)$ :

$$
P(n, T) Q(n, T) \Psi(n, z)=q(z) \Psi(n, z) .
$$

We will give a version of Theorem 1.2 from $[3]$ which provides general sufficient conditions on the operators $P(n, T)$ and $Q(n, T)$ under which $\Psi(n, z)$ is also an eigenfunction of a differential operator in the variable $z$. (The original result of $[\mathbf{3}]$ deals with "bispectral" Darboux transformations in an arbitrary associative algebra but in the form to be used, needs an additional refinement.)

Theorem 4.1. Assume that the operator $q\left(L_{\alpha, \beta ; \varepsilon}(n, T)\right) \in \mathcal{A}_{\alpha, \beta ; \varepsilon}$ is factorized as

$$
q\left(L_{\alpha, \beta ; \varepsilon}(n, T)\right)=\left(Q_{0}(n, T) \nu(n)^{-1}\right)\left(\mu(n)^{-1} P_{0}(n, T)\right)
$$

for some difference operators $P_{0}(n, T), Q_{0}(n, T) \in \mathcal{B}_{\alpha, \beta ; \varepsilon}$ and rational functions $\mu(n), \nu(n) \in \mathcal{K}_{\alpha, \beta ; \varepsilon}$, such that the coefficients of the operators $\mu(n)^{-1} P_{0}(n, T), Q_{0}(n, T) \nu(n)^{-1}$ are correctly defined for $n \in \mathbb{Z}$. Then the function

$$
\Psi(n, z)=\left(\mu^{-1}(n) P_{0}(n, T)\right) p_{\varepsilon}^{\alpha, \beta}(n, z)
$$

satisfies the relations

$$
\begin{aligned}
& \left(\mu(n)^{-1} P_{0}(n, T)\right)\left(Q_{0}(n, T) \nu(n)^{-1}\right) \Psi(n, z)=q(z) \Psi(n, z), \\
& b\left(P_{0}\right)\left(z, \partial_{z}\right) b\left(Q_{0}\right)\left(z, \partial_{z}\right) q(z)^{-1} \Psi(n, z)=\mu(n) \nu(n) \Psi(n, z),
\end{aligned}
$$

i.e., it is bispectral.

Note that in Theorem 4.1 we do not assume that the rational functions $\mu(n)^{-1}$ and $\nu(n)^{-1}$ are well-defined for $n \in \mathbb{Z}$, but only that the "ratios" $\mu(n)^{-1} P_{0}(n, T)$ and $Q_{0}(n, T) \nu(n)^{-1}$ are. Because of this a small modification of the original proof from $[\mathbf{3}]$ is necessary. 
First of all since the algebra $\mathcal{B}_{\alpha, \beta ; \varepsilon}^{\prime}$ has no zero divisors, Equation (4.14) implies (see [3])

$$
(b \nu)\left(z, \partial_{z}\right)(b \mu)\left(z, \partial_{z}\right)=\left(b P_{0}\right)\left(z, \partial_{z}\right) q(z)^{-1}\left(b Q_{0}\right)\left(z, \partial_{z}\right) .
$$

For all values of $n$ for which $\mu(n)$ does not vanish we have

$$
\Psi(n, z)=\mu(n)^{-1}\left(b P_{0}\right)\left(z, \partial_{z}\right) p_{\varepsilon}^{\alpha, \beta}(n, z)
$$

and (4.17) holds, as a consequence of (4.18). The validity of (4.17) for all $n \in \mathbb{Z}$ follows from the definition (4.15) of $\Psi(n, z)$ and the fact that $p_{\varepsilon}^{\alpha, \beta}(n, z)$ has an expansion in $z$ around $z=1$ with coefficients that are rational functions in $n$ (recall (3.12)).

Returning to the sets $\mathcal{D}_{\alpha, \beta ; \varepsilon}^{(k, l)}$ of Darboux transformations from the operators $L_{\alpha, \beta ; \varepsilon}(n, T)$, we need to find which of the operators $P(n, T)$ from Equation (3.30) can be expressed in the form $\mu(n)^{-1} P_{0}(n, T)$ with $\mu(n)$ and $P_{0}(n, T)$ as above. According to Theorem 4.1 the corresponding operators $L(n, T) \in \mathcal{D}_{\alpha, \beta ; \varepsilon}^{(k, l)}$ will be bispectral with bispectral eigenfunction (3.34) (see also (4.15)). For this we need an invariant description of the linear space of difference operators

$$
\begin{array}{r}
\mathcal{R}_{\alpha, \beta ; \varepsilon}=\operatorname{Span}\left\{\mu(n)^{-1} S(n, T) \mid S(n, T) \in \mathcal{B}_{\alpha, \beta ; \varepsilon}, \mu(n) \in \mathcal{K}_{\alpha, \beta ; \varepsilon},\right. \\
\text { such that } \left.\mu(n)^{-1} S(n, T) \text { is well-defined for } n \in \mathbb{Z}\right\} .
\end{array}
$$

This will be obtained in the next subsection. Here we would like to note that the dual object - the linear space of differential operators

$$
\mathcal{R}_{\alpha, \beta ; \varepsilon}^{\prime}=\operatorname{Span}\left\{g(z)^{-1} G\left(z, \partial_{z}\right) \mid G\left(z, \partial_{z}\right) \in \mathcal{B}_{\alpha, \beta ; \varepsilon}^{\prime}, g(z) \in \mathcal{K}_{\alpha, \beta ; \varepsilon}^{\prime}\right\}
$$

is much easier to describe. It is just the space of differential operators with rational coefficients. This is a consequence of the fact that the commutator

$$
\left[B_{\alpha, \beta}\left(z, \partial_{z}\right), z\right]=2\left(z^{2}-1\right) \partial_{z}+((\alpha-\beta)+(\alpha+\beta+2) z)
$$

is a first order differential operator that belongs to $\mathcal{B}_{\alpha, \beta ; \varepsilon}^{\prime}$ and $z \in \mathcal{K}_{\alpha, \beta ; \varepsilon}^{\prime}$ (see Equation (4.10)). Unfortunately for our proof of the fact that the operators from $\mathcal{D}_{\alpha, \beta ; \varepsilon}^{(k, l)}$ are bispectral we need the space $\mathcal{R}_{\alpha, \beta ; \varepsilon}$, and not the space $\mathcal{R}_{\alpha, \beta ; \varepsilon}^{\prime}$.

\subsection{Description of $\mathcal{R}_{\alpha, \beta ; \varepsilon}$. Denote by $\Delta$ the abstract algebra of difference} operators $M(n, T)$ with rational coefficients; that is the algebra over $\mathbb{C}$, generated by rational functions in $n$, the shift operator $T$, and its inverse $T^{-1}$, subject to the relation

$$
T h(n)=h(n+1) T, \text { for all rational functions } h(n) .
$$

Here we do not require that the coefficients of an operator $M(n, T)$ in $\Delta$ be well-defined for $n \in \mathbb{Z}$. More explicitly these coefficients could have poles at some $n \in \mathbb{Z}$. The subspace of $\Delta$ consisting of operators having this extra regularity property will be denoted by $\Delta^{\text {reg. }}$. We will identify the space of 
difference operators with rational coefficients acting on functions $f: \mathbb{Z} \rightarrow \mathbb{C}$ with $\Delta^{\text {reg }}$. In particular, $\widetilde{\mathcal{B}}_{\alpha, \beta ; \varepsilon} \subset \widetilde{\mathcal{R}}_{\alpha, \beta ; \varepsilon} \subset \Delta^{\mathrm{reg}}$.

Define an involution $I$ in the algebra $\Delta$ acting on rational functions $h(n)$ by

$$
(I h)(n)=h(-(n+2 \varepsilon+\alpha+\beta+1))
$$

and on the shift operator $T$ by

$$
I(T)=T^{-1} .
$$

The involution $I$ is correctly defined since

$$
I(T)(I h)(n)=(I h)(n+1) I(T) .
$$

Denote the fixed points of $I$ in $\Delta$ by $\Delta^{I}$ :

$$
\Delta^{I}=\{M(n, T) \in \Delta \mid I(M(n, T))=M(n, T)\} .
$$

Let

$$
\phi(n)=\frac{(\varepsilon+\alpha+1)_{n}}{(\varepsilon+1)_{n}}
$$

(cf. the definition (2.8) of $p_{\varepsilon}^{\alpha, \beta}(n, z)$ for $\alpha \notin \mathbb{Z}_{<0}$ ).

Theorem 4.2. The space of difference operators $\mathcal{R}_{\alpha, \beta ; \varepsilon}$ defined in (4.19) is characterized by

$$
\mathcal{R}_{\alpha, \beta ; \varepsilon}=\operatorname{Ad}_{\phi(n)}\left(\Delta^{I} \cap \Delta^{\mathrm{reg}}\right),
$$

i.e., after conjugation by $\phi(n)^{-1}$ all operators from $\widetilde{\mathcal{R}}_{\alpha, \beta ; \varepsilon}$ are I-invariant. Proof. Consider first the case $\alpha \notin \mathbb{Z}_{<0}$. Let

$$
\widetilde{p}_{\varepsilon}^{\alpha, \beta}(n, z)=\phi(n)^{-1} p_{\varepsilon}^{\alpha, \beta}(n, z) .
$$

The expression (2.8) implies

$$
\widetilde{p}_{\varepsilon}^{\alpha, \beta}(n, z)=F(-(-n+\varepsilon), n+\varepsilon+\alpha+\beta+1 ; \alpha+1 ;(1-z) / 2) .
$$

Let $\widetilde{\mathcal{B}}_{\alpha, \beta ; \varepsilon}, \widetilde{\mathcal{B}}_{\alpha, \beta ; \varepsilon}^{\prime}, \widetilde{\mathcal{K}}_{\alpha, \beta ; \varepsilon}, \widetilde{\mathcal{K}}_{\alpha, \beta ; \varepsilon}^{\prime}, \widetilde{\mathcal{R}}_{\alpha, \beta ; \varepsilon}$, and $\widetilde{\mathcal{R}}_{\alpha, \beta ; \varepsilon}^{\prime}$, denote the $\mathcal{B}, \mathcal{K}$ and $\mathcal{R}$ objects associated with the functions $\widetilde{p}_{\varepsilon}^{\alpha, \beta}(n, z)$ (see the beginning of Section 4.1 and Equations (4.6), (4.7), (4.19) and (4.20) for the appropriate definitions). Obviously

$$
\widetilde{\mathcal{R}}_{\alpha, \beta ; \varepsilon}=\operatorname{Ad}_{\phi(n)} \mathcal{R}_{\alpha, \beta ; \varepsilon}, \widetilde{\mathcal{B}}_{\alpha, \beta ; \varepsilon}=\operatorname{Ad}_{\phi(n)} \mathcal{B}_{\alpha, \beta ; \varepsilon},
$$

and $\widetilde{\mathcal{K}}_{\alpha, \beta ; \varepsilon}=\mathcal{K}_{\alpha, \beta ; \varepsilon}, \widetilde{\mathcal{R}}_{\alpha, \beta ; \varepsilon}^{\prime}=\mathcal{R}_{\alpha, \beta ; \varepsilon}^{\prime}, \widetilde{\mathcal{K}}_{\alpha, \beta ; \varepsilon}^{\prime}=\mathcal{K}_{\alpha, \beta ; \varepsilon}^{\prime}, \widetilde{\mathcal{B}}_{\alpha, \beta ; \varepsilon}^{\prime}=\mathcal{B}_{\alpha, \beta ; \varepsilon}^{\prime}$. In this notation, the statement of the theorem is equivalent to

$$
\widetilde{\mathcal{R}}_{\alpha, \beta ; \varepsilon}=\Delta^{I} \cap \Delta^{\mathrm{reg}} .
$$

To prove that the l.h.s. of (4.27) is contained in the r.h.s., let us fix an operator $\widetilde{R}(n, T) \in \widetilde{\mathcal{R}}_{\alpha, \beta ; \varepsilon}$. There exists a difference operator $\widetilde{S}(n, T) \in$ 
$\widetilde{\mathcal{B}}_{\alpha, \beta ; \varepsilon}$ and a function $\widetilde{\mu}(n) \in \widetilde{\mathcal{K}}_{\alpha, \beta ; \varepsilon}$ such that $\widetilde{R}(n, T)=\widetilde{\mu}(n)^{-1} \widetilde{S}(n, T)$. We will prove that all operators from $\widetilde{\mathcal{B}}_{\alpha, \beta ; \varepsilon}$ are $I$-invariant. This in particular shows that all functions from $\widetilde{\mathcal{K}}_{\alpha, \beta ; \varepsilon} \subset \widetilde{\mathcal{B}}_{\alpha, \beta ; \varepsilon}$ are $I$-invariant and so are all operators from $\widetilde{\mathcal{R}}_{\alpha, \beta ; \varepsilon}$.

If $\widetilde{S}(n, T) \in \widetilde{\mathcal{B}}_{\alpha, \beta ; \varepsilon}$, then there exists a differential operator $G\left(z, \partial_{z}\right)$ for which

$$
\widetilde{S}(n, T) \widetilde{p}_{\varepsilon}^{\alpha, \beta}(n, z)=G\left(z, \partial_{z}\right) \widetilde{p}_{\varepsilon}^{\alpha, \beta}(n, z) .
$$

The fact that the hypergeometric function $F(a, b ; c ; x)$ is symmetric with respect to $a$ and $b$, and formula (4.26) for $\widetilde{p}_{\varepsilon}^{\alpha, \beta}(n, z)$ imply

$$
I(\widetilde{S}(n, T)) \widetilde{p}_{\varepsilon}^{\alpha, \beta}(n, z)=G\left(z, \partial_{z}\right) \widetilde{p}_{\varepsilon}^{\alpha, \beta}(n, z) .
$$

Combining (4.28) and (4.29), we conclude that

$$
(\widetilde{S}(n, T)-I(\widetilde{S}(n, T))) \widetilde{p}_{\varepsilon}^{\alpha, \beta}(n, z)=0 .
$$

This is only possible if

$$
I(\widetilde{S}(n, T))=\widetilde{S}(n, T) .
$$

The harder part of the proof of (4.27) is to show that any $I$-invariant difference operator from $\Delta^{\text {reg }}$ belongs to $\widetilde{\mathcal{R}}_{\alpha, \beta ; \varepsilon}$. It is sufficient to prove that for any $\widetilde{R}(n, T) \in \Delta^{I}$ there exists $\widetilde{S}(n, T) \in \widetilde{\mathcal{B}}_{\alpha, \beta ; \varepsilon}$ and $\widetilde{\mu}(n) \in \widetilde{\mathcal{K}}_{\alpha, \beta ; \varepsilon}$ such that

$$
\widetilde{R}(n, T)=\widetilde{\mu}(n)^{-1} \widetilde{S}(n, T) .
$$

First let us write formulas (2.10) and (2.11) in terms of $\widetilde{p}_{\varepsilon}^{\alpha, \beta}(n, z)$. Equation (2.11) remains unchanged:

$$
\lambda_{\varepsilon}(n) \widetilde{p}_{\varepsilon}^{\alpha, \beta}(n, z)=B_{\alpha, \beta}\left(z, \partial_{z}\right) \widetilde{p}_{\varepsilon}^{\alpha, \beta}(n, z),
$$

while Equation (2.10) becomes

$$
\widetilde{L}_{\alpha, \beta ; \varepsilon}(n, T) \widetilde{p}_{\varepsilon}^{\alpha, \beta}(n, z)=z \widetilde{p}_{\varepsilon}^{\alpha, \beta}(n, z)
$$

with

$$
\begin{aligned}
& \widetilde{L}_{\alpha, \beta ; \varepsilon}(n, T)=\phi(n)^{-1} L_{\alpha, \beta ; \varepsilon}(n, T) \phi(n) \\
& =\frac{2(n+\varepsilon+\alpha+1)(n+\varepsilon+\alpha+\beta+1)}{(2 n+2 \varepsilon+\alpha+\beta+1)(2 n+2 \varepsilon+\alpha+\beta+2)} T \\
& \quad+\frac{\alpha^{2}-\beta^{2}}{(2 n+2 \varepsilon+\alpha+\beta)(2 n+2 \varepsilon+\alpha+\beta+2)} \\
& \quad+\frac{2(n+\varepsilon)(n+\varepsilon+\beta)}{(2 n+2 \varepsilon+\alpha+\beta)(2 n+2 \varepsilon+\alpha+\beta+1)} T^{-1} .
\end{aligned}
$$


The algebra $\Delta$ has a natural $\mathbb{Z}_{\geq 0}$ filtration where $\Delta_{d}$ consists of all operators from $\Delta$ with support $[-d, d]$. Denote $\Delta_{d}^{I}=\Delta^{I} \cap \Delta_{d}$.

We will prove that any difference operator $R_{d}^{I}(n, T) \in \Delta_{d}^{I}$, can be decomposed as a sum

$$
R_{d}^{I}(n, T)=\widetilde{\mu}(n)^{-1} \widetilde{S}(n, T)+R_{d-1}^{I}(n, T)
$$

where

$$
\begin{aligned}
& \widetilde{S}(n, T) \in \widetilde{\mathcal{B}}_{\alpha, \beta ; \varepsilon}, \widetilde{\mu}(n) \in \widetilde{\mathcal{K}}_{\alpha, \beta ; \varepsilon}, \\
& R_{d-1}^{I}(n, T) \in \Delta_{d-1}^{I} .
\end{aligned}
$$

Since $\Delta_{0}^{I}=\mathbb{C}\left(\lambda_{e}(n)\right)$ (any $I$-invariant rational function in $n$ is a rational function in $\left.\lambda_{e}(n)\right)$, by induction on $d$ Equation (4.33) implies that

$$
R_{d}^{I}(n, T) \in \widetilde{\mathcal{R}}_{\alpha, \beta ; \varepsilon} .
$$

A straightforward computation yields

$$
\begin{aligned}
\operatorname{ad}_{\lambda_{\varepsilon}(n)} T^{d} & =\left(\lambda_{\varepsilon}(n)-\lambda_{\varepsilon}(n+d)\right) T^{d} \\
& =-d(2 n+2 \varepsilon+\alpha+\beta+d+1) T^{d},
\end{aligned}
$$

and thus

$$
\begin{aligned}
& \operatorname{ad}_{\lambda_{\varepsilon}(n)}\left(\operatorname{ad}_{\lambda_{\varepsilon}(n)}+1\right) \widetilde{L}_{\alpha, \beta ; \varepsilon} \\
& =2(\operatorname{Id}+I)((n+\varepsilon+\alpha+1)(n+\varepsilon+\alpha+\beta+1) T) .
\end{aligned}
$$

So

$$
\begin{aligned}
& \left(\operatorname{ad}_{\lambda_{\varepsilon}(n)}\left(\operatorname{ad}_{\lambda_{\varepsilon}(n)}+1\right) \widetilde{L}_{\alpha, \beta ; \varepsilon}\right)^{d} \\
& =2^{d}(\operatorname{Id}+I)\left(\prod_{i=1}^{d}(n+\varepsilon+\alpha+i)(n+\varepsilon+\alpha+\beta+i) T^{d}\right)+U_{d-1}
\end{aligned}
$$

for some $U_{d-1} \in \Delta_{d-1}^{I}$. (Here we use the $I$-invariance of $L_{\alpha, \beta ; \varepsilon}(n, T)$.) Denote for simplicity

$$
c_{d}(n)=2^{d} \prod_{i=1}^{d}((n+\varepsilon+\alpha+i)(n+\varepsilon+\alpha+\beta+i))
$$

and let

$$
R_{d}^{I}(n, T)=\sum_{i=-d}^{d} \frac{a_{i}(n)}{b_{i}(n)} T^{i}, a_{i}(n), b_{i}(n) \in \mathbb{C}[n] .
$$


Using (4.36) and (4.37) we obtain

$$
\begin{aligned}
& a_{d}\left(-\frac{1}{2 d} \operatorname{ad}_{\lambda_{\varepsilon}(n)}-\frac{\alpha+\beta+d+1}{2}-\varepsilon\right) \\
& \quad \cdot b_{d}\left(\frac{1}{2 d} \operatorname{ad}_{\lambda_{\varepsilon}(n)}-\frac{\alpha+\beta-d+1}{2}-\varepsilon\right) \\
& \quad \cdot c_{d}\left(\frac{1}{2 d} \operatorname{ad}_{\lambda_{\varepsilon}(n)}-\frac{\alpha+\beta-d+1}{2}-\varepsilon\right)\left(\operatorname{ad}_{\lambda_{\varepsilon}(n)}\left(\operatorname{ad}_{\lambda_{\varepsilon}(n)}+1\right) \widetilde{L}_{\alpha, \beta ; \varepsilon}\right)^{d} \\
& =(\operatorname{Id}+I)\left(b_{d}(n)\left(I b_{d}\right)(n) c_{d}(n)\left(I c_{d}\right)(n) \frac{a_{d}(n)}{b_{d}(n)} T^{d}\right)+U_{d-1}
\end{aligned}
$$

for some other $U_{d-1} \in \Delta_{d-1}^{I}$. There exists a polynomial $q_{d}(n)$ for which

$$
b_{d}(n)\left(I b_{d}\right)(n) c_{d}(n)\left(I c_{d}\right)(n)=q_{d}\left(\lambda_{\varepsilon}(n)\right)
$$

because the polynomial in the l.h.s. is clearly $I$-invariant. Denote by $\widetilde{S}(n, T)$ the difference operator in (4.38). The l.h.s. of (4.38) implies that $\widetilde{S}(n, T)$ belongs to $\widetilde{\mathcal{B}}_{\alpha, \beta ; \varepsilon}$ and the r.h.s. implies

$$
R_{d}^{I}(n, T)-\left(q\left(\lambda_{\varepsilon}(n)\right)\right)^{-1} \widetilde{S}(n, T) \in \Delta_{d-1}^{I}
$$

which completes the proof of Theorem 4.2.

Remark 4.3. Any fuction $\widetilde{\mu}(n) \in \widetilde{\mathcal{K}}_{\alpha, \beta ; \varepsilon}$ is $I$-invariant and therefore is a rational function in $\lambda_{\varepsilon}(n)$. In fact $\widetilde{\mu}(n)$ should be a polynomial in $\lambda_{\varepsilon}(n)$. Indeed if $\widetilde{\mu}(n)=p\left(\lambda_{\varepsilon}(n)\right) / q\left(\lambda_{\varepsilon}(n)\right)$ for two polynomials $p(x), q(x) \in \mathbb{C}[x]$ such that $q(x) \chi p(x)$, then there exists a differential operator $G\left(z, \partial_{z}\right)$ with rational coefficients such that

$$
G\left(z, \partial_{z}\right) \widetilde{p}_{\varepsilon}^{\alpha, \beta}(n, z)=\frac{p\left(\lambda_{\varepsilon}(n)\right)}{q\left(\lambda_{\varepsilon}(n)\right)} \widetilde{p}_{\varepsilon}^{\alpha, \beta}(n, z)
$$

which implies

$$
p\left(B_{\alpha, \beta}\left(z, \partial_{z}\right)\right)=G\left(z, \partial_{z}\right) q\left(B_{\alpha, \beta}\left(z, \partial_{z}\right)\right) .
$$

This is impossible; if $z_{0}$ is a root of $q(x)$ and $p(x)$ of multiplicities $d_{1}>d_{2}$, then there exist a holomorphic function $g(z)$ in a domain $\Omega \subset \mathbb{C}$ such that

$$
\left(B_{\alpha, \beta}\left(z, \partial_{z}\right)-z_{0}\right)^{d_{1}} g(z)=0 \quad \text { and } \quad q\left(B_{\alpha, \beta}\left(z, \partial_{z}\right)-z_{0}\right) g(z) \neq 0
$$

which contradicts with (4.39). Since $\mathcal{K}_{\alpha, \beta ; \varepsilon}=\widetilde{\mathcal{K}}_{\alpha, \beta ; \varepsilon}$ we finally obtain

$$
\begin{aligned}
& \mathcal{K}_{\alpha, \beta ; \varepsilon}=\mathbb{C}\left[\lambda_{e}(n)\right], \\
& \mathcal{A}_{\alpha, \beta ; \varepsilon}^{\prime}=\mathbb{C}\left[B_{\alpha, \beta}\left(z, \partial_{z}\right)\right],
\end{aligned}
$$

as promised following (4.12) and (4.13). 
Remark 4.4. The second order bispectral differential operators of the even case of Duistermaat-Grünbaum's classification [7] are obtained as Darboux transformations from the Bessel operators

$$
L_{k}\left(x, \partial_{x}\right)=\partial_{x}^{2}-\frac{k(k-1)}{x^{2}}, k \in \mathbb{Z}+\frac{1}{2}
$$

in the sense of (3.1). More precisely for each operator $L\left(x, \partial_{x}\right)$ of this family there exists a differential operator with rational coefficients $P\left(x, \partial_{x}\right)$ such that

$$
L\left(x, \partial_{x}\right) P\left(x, \partial_{x}\right)=P\left(x, \partial_{x}\right) L_{k}\left(x, \partial_{x}\right) .
$$

In addition, the operator $P\left(x, \partial_{x}\right)$ satisfies

$$
P\left(x, \partial_{x}\right)=P\left(-x,-\partial_{x}\right) .
$$

Let $\mathcal{I}$ denote the involution of the algebra of differential operators with rational coefficients induced by the diffeomorphism $x \mapsto-x$ of $\mathbb{C}$ (i.e., $\left.(\mathcal{I} S)\left(x, \partial_{x}\right)=S\left(-x,-\partial_{x}\right)\right)$. Then (4.40) means that $P\left(x, \partial_{x}\right)$ is invariant under $\mathcal{I}$. This gives the relation of the approach of this paper via the involution $I$ and the space $\mathcal{R}_{\alpha, \beta ; \varepsilon}$ to the construction of [7].

\section{Bispectrality of $\mathcal{D}_{\alpha, \beta ; \varepsilon}^{(k, l)}$.}

In this section we prove our main result: When the parameters $\alpha$ and $\beta$ are subject to certain natural integrality conditions, the difference operators from $\mathcal{D}_{\alpha, \beta ; \varepsilon}^{(k, l)}$ are bispectral. As an example, for each $L(n, T) \in \mathcal{D}_{2,0 ; \varepsilon}^{(2,0)}$ we find a dual differential operator of order 10 .

The conditions (2.13) on $\alpha, \beta, \varepsilon$ are assumed throughout this section.

5.1. Proof of the main result. The conjugation by the function $\phi(n)$ (see (4.23)), used in Theorem 4.2, leads us to consider the functions $\Phi_{ \pm}^{(i)}:=$ $\varphi_{ \pm}^{(i)}(n) / \phi(n), \Psi_{ \pm}^{(i)}:=\psi_{ \pm}^{(i)}(n) / \phi(n)$. Because of Equations (3.18)-(3.21) they are explicitly given by the formulas

$$
\Phi_{+}^{(i)}(n)=\frac{(-(n+\varepsilon))_{i}(n+\varepsilon+\alpha+\beta+1)_{i}}{(\alpha+1)_{i}(-2)^{i}},
$$

$$
\Psi_{+}^{(i)}(n)=\frac{(\varepsilon+\beta+1)_{n}(\varepsilon+1)_{n}}{(\varepsilon+\alpha+1)_{n}(\varepsilon+\alpha+\beta+1)_{n}} \frac{(-(n+\varepsilon+\alpha+\beta))_{i}(n+\varepsilon+1)_{i}}{(-\alpha+1)_{i}(-2)^{i}},
$$

$$
\Phi_{-}^{(i)}(n)=\frac{(\varepsilon+\beta+1)_{n}}{(-1)^{n}(\varepsilon+\alpha+1)_{n}} \frac{(-(n+\varepsilon))_{i}(n+\varepsilon+\alpha+\beta+1)_{i}}{(\beta+1)_{i} 2^{i}},
$$


$(5.4)$

$$
\Psi_{-}^{(i)}(n)=\frac{(\varepsilon+1)_{n}}{(-1)^{n}(\varepsilon+\alpha+\beta+1)_{n}} \frac{(-(n+\varepsilon+\alpha+\beta))_{i}(n+\varepsilon+1)_{i}}{(-\beta+1)_{i} 2^{i}} .
$$

Lemma 5.1. If $\alpha \in \mathbb{Z}$, then for $i \leq|\alpha|-1, \Phi_{+}^{(i)}(n)$ and $\Psi_{+}^{(i)}(n)$ are $I$ invariant rational functions of $n$.

If $\alpha \in \mathbb{Z}$ and $\beta \in \mathbb{Z}$ then for $i \leq|\alpha|-1, j \leq|\beta|-1, \Phi_{+}^{(i)}(n), \Psi_{+}^{(i)}(n)$, $(-1)^{n} \Phi_{-}^{(j)}(n)$, and $(-1)^{n} \Psi_{-}^{(j)}(n)$ are rational functions of $n, \Phi_{+}^{(i)}(n), \Psi_{+}^{(i)}(n)$ are $I$-invariant, and

$$
\begin{aligned}
& I\left((-1)^{n} \Phi_{-}^{(j)}(n)\right)=(-1)^{\alpha+\beta}\left((-1)^{n} \Phi_{-}^{(j)}(n)\right), \\
& I\left((-1)^{n} \Psi_{-}^{(j)}(n)\right)=(-1)^{\alpha+\beta}\left((-1)^{n} \Psi_{-}^{(j)}(n)\right) .
\end{aligned}
$$

Proof. First note that

$$
\begin{aligned}
& (-(n+\varepsilon))_{i}(n+\varepsilon+\alpha+\beta+1)_{i} \\
& =\prod_{r=0}^{i-1}(-(n+\varepsilon)+r)(n+\varepsilon+\alpha+\beta+1+r) \\
& =(-1)^{k} \prod_{r=0}^{i-1}(\lambda(n)-r(\alpha+\beta+1+r))
\end{aligned}
$$

and similarly

$$
(-(n+\varepsilon+\alpha+\beta))_{i}(n+\varepsilon+1)_{i}=(-1)^{i} \prod_{r=0}^{i-1}(\lambda(n)-(\alpha+\beta-r)(r+1))
$$

are $I$-invariant polynomials in $n$.

To prove the first statement of the lemma we use a similar computation. Restricting to the case $\alpha \in \mathbb{Z}_{>0}$ :

$$
\begin{aligned}
& \frac{(\varepsilon+\beta+1)_{n}(\varepsilon+1)_{n}}{(\varepsilon+\alpha+1)_{n}(\varepsilon+\alpha+\beta+1)_{n}} \\
& =\frac{(\varepsilon+1)_{\alpha}(\varepsilon+\beta+1)_{\alpha}}{(n+\varepsilon+1)_{\alpha}(n+\varepsilon+\beta+1)_{\alpha}} \\
& =\frac{(\varepsilon+1)_{\alpha}(\varepsilon+\beta+1)_{\alpha}}{\prod_{r=1}^{\alpha}(n+\varepsilon+r)(n+\varepsilon+\beta+\alpha+1-r)} \\
& =\frac{(\varepsilon+1)_{\alpha}(\varepsilon+\beta+1)_{\alpha}}{\prod_{r=1}^{\alpha}(\lambda(n)+r(\alpha+\beta+1-r))} .
\end{aligned}
$$

The proof of the second statement is analogous. Assuming $\alpha, \beta \in \mathbb{Z}_{>0}$ and $\beta \geq \alpha$ we obtain

$$
\frac{(\varepsilon+\beta+1)_{n}}{(\varepsilon+\alpha+1)_{n}}=\frac{(\varepsilon+\alpha+n+1)_{\beta-\alpha}}{(\varepsilon+\alpha+1)_{\beta-\alpha}}
$$




$$
\begin{aligned}
& =\frac{q(n) \prod_{r=1}^{\left[\frac{\beta-\alpha}{2}\right]}(n+\varepsilon+\alpha+r)(n+\varepsilon+\beta+1-r)}{(\varepsilon+\alpha+1)_{\beta-\alpha}} \\
& =\frac{q(n) \prod_{r=1}^{\left[\frac{\beta-\alpha}{2}\right]}(\lambda(n)+(\alpha+r)(\beta+1-r))}{(\varepsilon+\alpha+1)_{\beta-\alpha}}
\end{aligned}
$$

with

$$
q(n)=\left\{\begin{array}{ll}
1, & \text { if } \beta+\alpha \text { is even } \\
n+\varepsilon+(\alpha+\beta+1) / 2, & \text { if } \beta+\alpha \text { is odd }
\end{array} .\right.
$$

(Since $\alpha \in \mathbb{Z}$, the first condition is equivalent to $2 \mid(\beta-\alpha)$ and the second one to $2 \chi(\beta-\alpha)$.) To finish the proof of (5.5) we just observe that

$$
I(n+\varepsilon+(\alpha+\beta+1) / 2)=-(n+\varepsilon+(\alpha+\beta+1) / 2) .
$$

The remaining cases for $\alpha, \beta \in \mathbb{Z}$ are treated analogously.

The identity (5.6) follows from the analogous formula

$$
\frac{(\varepsilon+1)_{n}}{(\varepsilon+\alpha+\beta+1)_{n}}=\frac{(\varepsilon+1)_{\alpha+\beta}}{q(n) \prod_{r=1}^{\left[\frac{\beta+\alpha}{2}\right]}(\lambda(n)+r(\alpha+\beta+1-r))}
$$

and Equation (5.7).

Throughout this proof, for a real number $x$ by $[x]$ we denote its integer part.

Theorem 5.2. Assuming (2.13), the following sets consist of bispectral difference operators:

1) $\mathcal{D}_{\alpha, \beta ; \varepsilon}^{(k, 0)}$ if $\alpha \in \mathbb{Z}$ and $k \leq|\alpha|$,

2) $\mathcal{D}_{\alpha, \beta ; \varepsilon}^{(0, l)}$ if $\beta \in \mathbb{Z}$ and $l \leq|\beta|$,

3) $\mathcal{D}_{\alpha, \beta ; \varepsilon}^{(k, \ell)}$ if $\alpha, \beta \in \mathbb{Z}$ and $k \leq|\alpha|, l \leq|\beta|$.

When the conditions (2.13) are not met but the operator $L_{\alpha, \beta ; \varepsilon}(n, T)$ is still well-defined the arguments below can be adapted properly. We do not pursue that here.

Proof. Because of the relation (3.37) the second case follows from the first one.

Let us restrict to instances 1) and 3) of the theorem above. In each of them we can assume that $k+l$ is even using (3.38). Fix an operator $L(n, z) \in \mathcal{D}_{\alpha, \beta ; \varepsilon}^{(k, l)}$, determined by a choice of the functions $\left\{f^{(i)}(n)\right\}_{i=0}^{k+l-1}$, i.e., a choice of admissible values of the complex parameters $A, B, C, D$ (see Section 3.3). It has the eigenfunction $\Psi(n, z)$ defined in (3.34)

$$
L(n, T) \Psi(n, z)=z \Psi(n, z),
$$


cf. (3.35). We need to show that there exists a differential operator $B\left(z, \partial_{z}\right)$ having $\Psi(n, z)$ as an eigenfunction, that is

$$
B\left(z, \partial_{z}\right) \Psi(n, z)=\theta(n) \Psi(n, z)
$$

for some function $\theta(n)$.

Define the functions

$$
F^{(i)}(n)=f^{(i)}(n) / \phi(n), \quad i=0, \ldots, k+l-1 .
$$

Let us put $s:=(k+l) / 2$ and consider the operator

$$
\widetilde{P}(n, T)=(-1)^{n l}\left|\begin{array}{cccc}
F^{(0)}(n-s) & \ldots & F^{(k+l-1)}(n-s) & T^{-s} \\
\ldots & \ldots & \ldots & \ldots \\
F^{(0)}(n+s) & \ldots & F^{(k+l-1)}(n+s) & T^{s}
\end{array}\right| .
$$

It is a regular difference operator with kernel given by $\operatorname{Span}\left\{F^{(i)}(n)\right\}_{i=0}^{k+l-1}$. Hence it is related to the operator $P(n, T)$ (recall Equation (3.30)) by

$$
P(n, T)=d(n)^{-1} \phi(n)^{-1} T^{-s} \widetilde{P}(n, T) \phi(n)
$$

where

$$
d(n)=(-1)^{n l} \operatorname{det}\left(F^{(i)}(n+j)\right)_{i, j=0,-k-l}^{k+l-1,-1}
$$

is the leading coefficient of $T^{-s} \widetilde{P}(n, T)$. Lemma 5.1 implies that $F^{(0)}(n), \ldots$, $F^{(k-1)}(n)$, and $(-1)^{n} F^{(k)}(n), \ldots,(-1)^{n} F^{(k+l-1)}(n)$ are rational functions in $n$. This implies that for $i=k, \ldots, k+l-1$ and for all $j \in \mathbb{Z},(-1)^{n} F^{(i)}(n+$ $j$ ) are also rational functions in $n$ and thus $\widetilde{P}(n, T)$ has rational coefficients. In addition Lemma 5.1 gives

$$
I\left(F^{(i)}(n+j)\right)=F^{(i)}(n-j), i=0, \ldots, k-1, j \in \mathbb{Z}
$$

and

$$
\begin{aligned}
I\left((-1)^{n} F^{(i)}(n+j)\right)=(-1)^{(\alpha+\beta) / 2}\left((-1)^{n} F^{(i)}(n-j)\right), & \\
& i=k, \ldots, k+l-1, \quad j \in \mathbb{Z} .
\end{aligned}
$$

Taking into account that $I(T)=T^{-1}$ we obtain

$$
I(\widetilde{P}(n, T))=(-1)^{s}(-1)^{(\alpha+\beta) l} \widetilde{P}(n, T)
$$

where the factor $(-1)^{s}$ comes from exchanging the pairs of rows $(1, k+l+1)$, $\ldots,(s, s+2)$. Set

$$
q(n)= \begin{cases}1, & \text { if } s+(\alpha+\beta) l \text { is even } \\ (n+\varepsilon+(\alpha+\beta+1) / 2), & \text { if } s+(\alpha+\beta) l \text { is odd }\end{cases}
$$

and consider the operator

$$
\bar{P}(n, T)=q(n) \widetilde{P}(n, T) .
$$


Because of the conditions (2.13), $q(n)$ does not vanish for $n \in \mathbb{Z}$. Taking into account (5.9) one sees that $\bar{P}(n, T)$ is related to $P(n, T)$ by

$$
P(n, T)=\frac{d(n) \phi(n-s)}{q(n-s) \phi(n)} T^{-s} \phi(n)^{-1} \bar{P}(n, T) \phi(n) .
$$

Since $\bar{P}(n, T)$ is a regular difference operator and $\phi(n)$ does not vanish for $n \in \mathbb{Z}$ (recall (2.13)), there exists a difference operator with rational coefficients $\bar{Q}(n, T)$ such that

$$
\begin{aligned}
& \left(L_{\alpha, \beta ; \varepsilon}(n, T)-1\right)^{k}\left(L_{\alpha, \beta ; \varepsilon}(n, T)+1\right)^{l} \\
& =\left(\phi(n)^{-1} \bar{Q}(n, T) \phi(n)\right)\left(\phi(n)^{-1} \bar{P}(n, T) \phi(n)\right) .
\end{aligned}
$$

From Equations (5.10) and (5.11) it follows that $\bar{P}(n, T)$ is $I$-invariant. Finally combining this with the $I$-invariance of $\phi(n)^{-1} L_{\alpha, \beta ; \varepsilon}(n, T) \phi(n)=$ $\widetilde{L}_{\alpha, \beta ; \varepsilon}(n, T)$ (see (4.32)) implies the $I$-invariance of the operator $\bar{Q}(n, T)$. Theorem 4.2 now gives

$$
\phi(n)^{-1} \bar{P}(n, T) \phi(n), \phi(n)^{-1} \bar{Q}(n, T) \phi(n) \in \mathcal{R}_{\alpha, \beta ; \varepsilon} .
$$

Applying Theorem 4.1 we obtain that the function

$$
\bar{\Psi}(n, z)=\phi(n) \bar{P}(n, T) \phi(n)^{-1} p_{\varepsilon}^{\alpha, \beta}(n, z)
$$

is an eigenfunction of a differential operator $B\left(z, \partial_{z}\right)$

$$
B\left(z, \partial_{z}\right) \bar{\Psi}(n, z)=h\left(\lambda_{\varepsilon}(n)\right) \bar{\Psi}(n, z),
$$

for some polynomial $h(x)$. Because of (5.12) our original function $\Psi(n, z) \in$ $\mathcal{D}_{\alpha, \beta ; \varepsilon}^{(k, l)}$ is related to $\bar{\Psi}(n, z)$ by

$$
\Psi(n, z)=P(n, T) p_{\varepsilon}^{\alpha, \beta}(n, z)=\frac{d(n) \phi(n-s)}{q(n-s) \phi(n)} T^{-(k+l) / 2} \bar{\Psi}(n, z) .
$$

Equation (5.14) implies that $\Psi(n, z)$ is an eigenfunction of the same operator $B\left(z, \partial_{z}\right)$ with eigenvalue $T^{-(k+l) / 2} h\left(\lambda_{\varepsilon}(n)\right)$ :

$$
B\left(z, \partial_{z}\right) \Psi(n, z)=h\left(\lambda_{\varepsilon}(n-(k+l) / 2)\right) \Psi(n, z) .
$$

5.2. An example: The set $\mathcal{D}_{2,0, \varepsilon}^{(2,0)}$. In this final subsection we consider in detail the case $\alpha=2, \beta=0, k=2, l=0$ and use this example for two different purposes. First we give the reader a guided tour through the results in this paper: We start with the function $p_{\varepsilon}^{2,0}(n, z)$ from $(2.8)$, give the ingredients needed to build the difference operator $P(n, T)(3.30)$ and the corresponding eigenfunction $\Psi(n, z)$ (3.34), and end with a description of the strategy used in the construction of a differential operator in the variable $z$ giving a bispectral situation. The algebra of possible differential operators in $z$ contains some whose order is lower than the one resulting from 
this construction. We close this subsection with an explicit expression for the (essentially unique) bispectral operator of minimal order and material related to this operator.

The functions $\varphi_{+}^{(i)}(n)$ and $\psi_{+}^{(i)}(n)(i=0,1)$ from (3.18)-(3.19) are given by

$$
\begin{array}{ll}
\varphi_{+}^{(0)}(n)=\frac{(n+\varepsilon+1)_{2}}{\kappa}, & \varphi_{+}^{(1)}(n)=\frac{(n+\varepsilon)_{4}}{6 \kappa}, \\
\psi_{+}^{(0)}(n)=\frac{\kappa}{(n+\varepsilon+1)_{2}}, & \psi_{+}^{(1)}(n)=-\frac{\kappa}{2},
\end{array}
$$

where

$$
\kappa=(\varepsilon+1)(\varepsilon+2) .
$$

The conditions $(2.13)$ reduce to $\varepsilon \notin \mathbb{Z}$.

An element $L(n, T) \in \mathcal{D}_{2,0, \varepsilon}^{(2,0)}$ is determined by a choice of the functions

$$
\begin{aligned}
& f^{(0)}(n)=A_{0} \varphi_{+}^{(0)}(n)+B_{0} \psi_{+}^{(0)}(n), \\
& f^{(1)}(n)=A_{1} \varphi_{+}^{(0)}(n)+B_{1} \psi_{+}^{(0)}(n)+A_{0} \varphi_{+}^{(1)}(n)+B_{0} \psi_{+}^{(1)}(n),
\end{aligned}
$$

cf. Section 3.3. We will restrict to the generic case when $A_{0} \neq 0$. In this case we can assume that $A_{0}=1$ and $A_{1}=0$ by dividing $f^{(0)}(n)$ by $A_{0}$ and then subtracting from $f^{(1)}(n)$ the term $A_{1} f^{(0)}(n)$. Recall that $L(n, T)$ depends only on $\operatorname{Span}\left\{f^{(0)}(n), f^{(1)}(n)\right\}$. Once this space has been specified by the choice of $B_{0}, B_{1}$ we can build the difference operator $P(n, T)$ as in (3.30) and we get the eigenfunction $\Psi(n, z)$ of $L(n, T)$ from (3.34).

The theory developed in Sections 4 and 5 makes it convenient to introduce the difference operators $\widetilde{P}(n, T)$, see (5.8), and $\bar{P}(n, T)$, see (5.11), related to $P(n, T)$ by $(5.9)$ and $(5.12)$.

The main point in the proof of Theorem 5.2 is that the operator $\bar{P}(n, T)$ defined in (5.11) (see also (5.8)) is $I$-invariant and thus $\phi(n) \bar{P}(n, T) \phi(n)^{-1} \in$ $\mathcal{R}_{2,0 ; \varepsilon}$. This implies that the function

$$
\bar{\Psi}(n, z)=\phi(n) \bar{P}(n, T) \phi(n)^{-1} p_{\varepsilon}^{\alpha, \beta}(n, z)
$$

(see (5.13)) can be expressed as

$$
\bar{\Psi}(n, z)=\mu(n)^{-1} G\left(z, \partial_{z}\right) p_{\varepsilon}^{\alpha, \beta}(n, z)
$$

for some differential operator with rational coefficients $G\left(z, \partial_{z}\right)$ and some polynomial $\mu(n)$ (recall the definition (4.19) of $\mathcal{R}_{2,0 ; \varepsilon}$ ). Now any operator $B\left(z, \partial_{z}\right)$ that is a Darboux transformation from $h\left(B_{2,0}\left(z, \partial_{z}\right)\right)$ for some $h(x) \in \mathbb{C}[x]$ via the operator $G\left(z, \partial_{z}\right)$, i.e.,

$$
B\left(z, \partial_{z}\right) G\left(z, \partial_{z}\right)=G\left(z, \partial_{z}\right) h\left(B_{2,0}\left(z, \partial_{z}\right)\right)
$$

will satisfy

$$
B\left(z, \partial_{z}\right) \bar{\Psi}(n, z)=h\left(\lambda_{\varepsilon}(n)\right) \bar{\Psi}(n, z)
$$


(a differential analog of (3.3)). The function $\Psi(n, z)$ is related to $\bar{\Psi}(n, z)$ by (5.15) and is also an eigenfunction of $B\left(z, \partial_{z}\right)$ but with eigenvalue $h(\lambda(n-1))$

$$
B\left(z, \partial_{z}\right) \Psi(n, z)=h\left(\lambda_{\varepsilon}(n-1)\right) \Psi(n, z),
$$

see (5.16). Combined with (3.35)

$$
L(n, T) \Psi(n, z)=z \Psi(n, z)
$$

this gives the desired bispectral pair $\left(L(n, T), B\left(z, \partial_{z}\right)\right)$.

The $I$-invariance of the operator $\bar{P}(n, T)$ in this special case can be observed directly. Because of (5.17), (5.18) the functions $F^{(i)}(n)=$ $f^{(i)}(n) / \phi(n), i=0,1$, see (4.23), are given in terms of

$$
\lambda_{\varepsilon}(n)=(n+\varepsilon)(n+\varepsilon+3)
$$

by

$$
\begin{aligned}
& F^{(0)}(n)=1+\frac{B_{0} \lambda_{\varepsilon}(n)}{6 \kappa}, \\
& F^{(1)}(n)=\frac{B_{1} \lambda_{\varepsilon}(n)}{6 \kappa}+\frac{\kappa}{\left(\lambda_{\varepsilon}(n)+1\right)}\left(\frac{\kappa}{\left(\lambda_{\varepsilon}(n)+1\right)}-\frac{B_{0}}{2}\right) .
\end{aligned}
$$

The operator $\bar{P}(n, T)$ is given by

$$
\bar{P}(n, T)=(n+\varepsilon+3 / 2)\left|\begin{array}{ccc}
F^{(0)}(n-1) & F^{(1)}(n-1) & T^{-1} \\
F^{(0)}(n) & F^{(1)}(n) & 1 \\
F^{(0)}(n+1) & F^{(1)}(n+1) & T
\end{array}\right|
$$

and it is $I$-invariant because of the $I$-invariance of $\lambda_{\varepsilon}(n)$ and the skew invariance of the factor in front compensating the effect of the exchange of first and third row. An operator $G\left(z, \partial_{z}\right)$ satisfying (5.20) is generated from the proof of Theorem 4.2. It is of high order and the one of minimal order 10 has the following form

$$
\begin{aligned}
G\left(z, \partial_{z}\right)= & (z-1)^{6}(z+1)^{5} \partial_{z}^{10}+(z-1)^{5}(z+1)^{4}(57 z+7) \partial_{z}^{9} \\
& +4(z-1)^{4}(z+1)^{3}\left(311 z^{2}+68 z-43\right) \partial_{z}^{8} \\
& +\left(3 B_{0} \kappa^{2}(z-1)^{2}(z+1)^{2}\right. \\
& \left.+2\left(18793 z^{4}+5796 z^{3}-15734 z^{2}-3636 z+1501\right)\right) \partial_{z}^{7}+\cdots
\end{aligned}
$$

Theorem 4.1 guarantees that (5.21) is satisfied for some polynomial $h(x)$. It also generates such a polynomial but it is again of high order. The one of minimal order 5 is given by

$$
\begin{aligned}
h(x-2)= & x^{5}-5 x^{4}+\left(10 B_{0} \kappa^{2}+8\right) x^{3} \\
& -\left(30 B_{1} \kappa^{2}+20 B_{0} \kappa^{2}+4\right) x^{2}-15 B_{0}^{2} \kappa^{4} x .
\end{aligned}
$$


Given $G\left(z, \partial_{z}\right)$ Equation (5.21) determines the dual bispectral operator $B\left(z, \partial_{z}\right)$ of $L(n, T)$ of minimal order uniquely. It is given by

$$
\begin{aligned}
B( & \left., \partial_{z}\right) \\
= & (z-1)^{5}(z+1)^{5} \partial_{z}^{10}+50(z-1)^{4} z(z+1)^{4} \partial_{z}^{9} \\
& +5(z-1)^{3}(z+1)^{3}(11 z-5)(17 z+7) \partial_{z}^{8} \\
+ & 160(z-1)^{2}(z+1)^{2}\left(52 z^{3}-7 z^{2}-28 z+1\right) \partial_{z}^{7} \\
+ & \left(30 B_{0}^{2} \kappa^{4} z+120 B_{1} \kappa^{2}(z-1)+120 B_{0} \kappa^{2}\right) \partial_{z}^{6} \\
+ & \left(180 B_{0} \kappa^{2}(z-1)^{2} z(z+1)^{2}\right. \\
& \left.+240(z-1)^{2}\left(337 z^{3}+504 z^{2}+141 z-30\right)\right) \partial_{z}^{5} \\
+ & \left(-30 B_{1} \kappa^{2}(z-1)^{2}(z+1)^{2}++120 B_{0} \kappa^{2}(z-1)(z+1)\left(8 z^{2}-z-3\right)\right. \\
& \left.+120(z-1)^{2}\left(641 z^{2}+758 z+161\right)\right) \partial_{z}^{4} \\
+ & \left(-240 B_{1} \kappa^{2}(z-1) z(z+1)+240 B_{0} \kappa^{2}\left(7 z^{3}-3 z^{2}-7 z+1\right)\right. \\
& \left.+960(z-1)^{2}(26 z+19)\right) \partial_{z}^{3} \\
+ & \left(-60 B_{1} \kappa^{2}(z-1)(7 z+5)+120 B_{0} \kappa^{2}(2 z+1)(3 z-5)+1440(z-1)^{2}\right) \partial_{z}^{2} \\
- & \left(30 B_{0}^{2} \kappa^{4} z+120 B_{1} \kappa^{2}(z-1)+120 B_{0} \kappa^{2}\right) \partial_{z} .
\end{aligned}
$$

In the cases $k=1, l=0,1$ and $\varepsilon=0$ the dual bispectral operator of minimal order was determined in $[\mathbf{1 9}, \mathbf{2 7}]$.

\section{References}

[1] B. Bakalov, E. Horozov and M. Yakimov, Bispectral algebras of commuting ordinary differential operators, Comm. Math. Phys., 190(2) (1997), 331-373, MR 99c:34188, Zbl 0912.34065.

[2] Highest weight modules over the $W_{1+\infty}$ algebra and the bispectral problem, Duke Math. J., 93(1) (1998), 41-72, MR 99h:58077.

[3] _ General methods for constructing bispectral operators, Phys. Lett. A, 222(12) (1996), 59-66, MR 97i:58160, Zbl 0972.37545.

[4] Yu. Berest, Huygens' principle and the bispectral problem, in 'The bispectral problem (Montreal, PQ, 1997), 11-30, CRM Proc. Lecture Notes, 14, Amer. Math. Soc., Providence, RI, 1998, MR 99c:58154, Zbl 0897.35043.

[5] Yu. Berest and G. Wilson, Classification of rings of differential operators on affine curves, IMRN, 2 (1999), 105-109, MR 2000f:14025, Zbl 0957.14013.

[6] S. Bochner, Über Sturm-Liouvillesche polynomsysteme, Math. Z., 29 (1929), 730-736.

[7] J.J. Duistermaat and F.A. Grünbaum, Differential equations in the spectral parameter, Comm. Math. Phys., 103(2) (1986), 177-240, MR 88j:58106, Zbl 0625.34007.

[8] P. Etingof and A. Varchenko, Traces of intertwiners for quantum groups and difference equations, I, Duke Math. J., 104(3) (2000), 391-432, MR 2001k:17021. 
[9] G. Felder, Y. Markov, V. Tarasov and A. Varchenko, Differential equations compatible with KZ equations, Math. Phys. Anal. Geom., 3(2) (2000), 139-177, CMP 1797943.

[10] F.A. Grünbaum and L. Haine, A theorem of Bochner, revisited, in 'Algebraic aspects of integrable systems,' 143-172, Progr. Nonlinear Differential Equations Appl., 26, Birkhäuser Boston, Boston, MA, 1997, MR 98f:58103, Zbl 0868.35116.

[11] _, Associated polynomials, spectral matrices and the bispectral problem, Methods and Applications of Analysis, 6(2) (1999), 209-224, CMP 1803 891, Zbl 0956.33007.

[12] F.A. Grünbaum, L. Haine and E. Horozov, Some functions that generalize the Krall-Laguerre polynomials, J. Comput. Appl. Math., 106(2) (1999), 271-297, MR 2000k:33017, Zbl 0926.33007.

[13] L. Haine, Beyond the classical orthogonal polynomials, in 'The bispectral problem (Montreal, PQ, 1997),' 47-65, CRM Proc. Lecture Notes, 14, Amer. Math. Soc., Providence, RI, 1998, MR 99d:33008, Zbl 0943.33006.

[14] _ The Bochner-Krall problem: Some new perspectives, to appear in Proceedings of the NATO Workshop on Special Functions, Tempe, Arizona, 2000.

[15] L. Haine and P. Iliev, Commutative rings of difference operators and an adelic flag manifold, IMRN, 6 (2000), 281-323, MR 2001d:37109.

[16] A rational analog of the Krall polynomials, J. of Phys. A: Math. Gen., 34(11) (2001), 2445-2457.

[17] E. Horozov and T. Milanov, Fuchsian bispectral operators, Bull. Sci. Math., 126 (2002), 161-192.

[18] A. Kasman and M. Rothstein, Bispectral Darboux transformations: The generalized Airy case, Phys. D, 102(3-4) (1997), 159-176, MR 98g:33005, Zbl 0890.58095.

[19] J. Koekoek and R. Koekoek, Differential equations for generalized Jacobi polynomials, J. Comput. Appl. Math., 126(1-2) (2000), 1-31, MR 2001m:34016, Zbl 0970.33004.

[20] H.L. Krall, Certain differential equations for Tchebicheff polynomials, Duke Math. J. 4 (1938), 705-718.

[21] W. Magnus, F. Oberhettinger and R. Soni, Formulas and Theorems for the Special Functions of Mathematical Physics, Springer Verlag, New York, 1966, MR 38 \#1291, Zbl 0143.08502.

[22] D. Mumford, An algebro-geometric construction of commuting operators and of solutions to the Toda lattice equation, Korteweg deVries equation and related nonlinear equation, in 'Proceedings of the International Symposium on Algebraic Geometry (Kyoto Univ., Kyoto, 1977),' 115-153, Kinokuniya Book Store, Tokyo, 1978, MR 83j:14041, Zbl 0423.14007.

[23] P. van Moerbeke and D. Mumford, The spectrum of difference operators and algebraic curves, Acta Math., 143(1-2) (1979), 93-154, MR 80e:58028, Zbl 0502.58032.

[24] G. Wilson, Bispectral commutative ordinary differential operators, J. Reine Angew. Math., 442 (1993), 177-204, MR 94m:58180, Zbl 0781.34051.

[25] — Collisions of Calogero-Moser particles and an adelic Grassmannian, with an appendix by I.G. Macdonald, Invent. Math., 133(1) (1998), 1-41, MR 99f:58107, Zbl 0906.35089.

[26] P. Wright, Darboux transformations, algebraic subvarieties of Grassmann manifolds, commuting flows and bispectrality, Ph.D. Thesis, Univ. California, Berkeley, 1987. 
[27] A. Zhedanov, A method of constructing Krall's polynomials, J. Comput. Appl. Math., 107 (1999), 1-20, MR 2000m:33012, Zbl 0929.33008.

Received October 18, 2000 and revised January 3, 2001. The first author was partially supported by NSF grant DMS94-00097. The second author was partially supported by NSF grants DMS94-00097 and DMS96-03239.

Department of Mathematics

UNiversity of CALIFORNIA AT BeRKELEy

Berkeley, CA 94720

E-mail address: grunbaum@math.berkeley.edu

Department of Mathematics

CORnEll University

ITHACA, NY 14853

E-mail address: milen@math.cornell.edu 